Integrative Systems

\title{
Time-of-Day-Dependent Gating of the Liver-Spinal Axis Initiates an Anti-Inflammatory Reflex in the Rat
}

\author{
[DEva Soto-Tinoco, ${ }^{1}$ DEsteban Santacruz, ${ }^{1}$ María del Carmen Basualdo-Sigales, ${ }^{1}$ \\ (D)Natalí N. Guerrero-Vargas, ${ }^{2}$ and ${ }^{-}$Ruud M. Buijs ${ }^{1}$
}

\section{https://doi.org/10.1523/ENEURO.0463-20.2020}

${ }^{1}$ Departamento de Biología Celular y Fisiología, Instituto de Investigaciones Biomédicas, Universidad Nacional Autónoma de México, Ciudad de México 04510, México and ${ }^{2}$ Departamento de Anatomía, Facultad de Medicina, Universidad Nacional Autónoma de México, Ciudad de México 04510, México

\section{Visual Abstract}

\section{ZT2}

4) High Spinal sensing

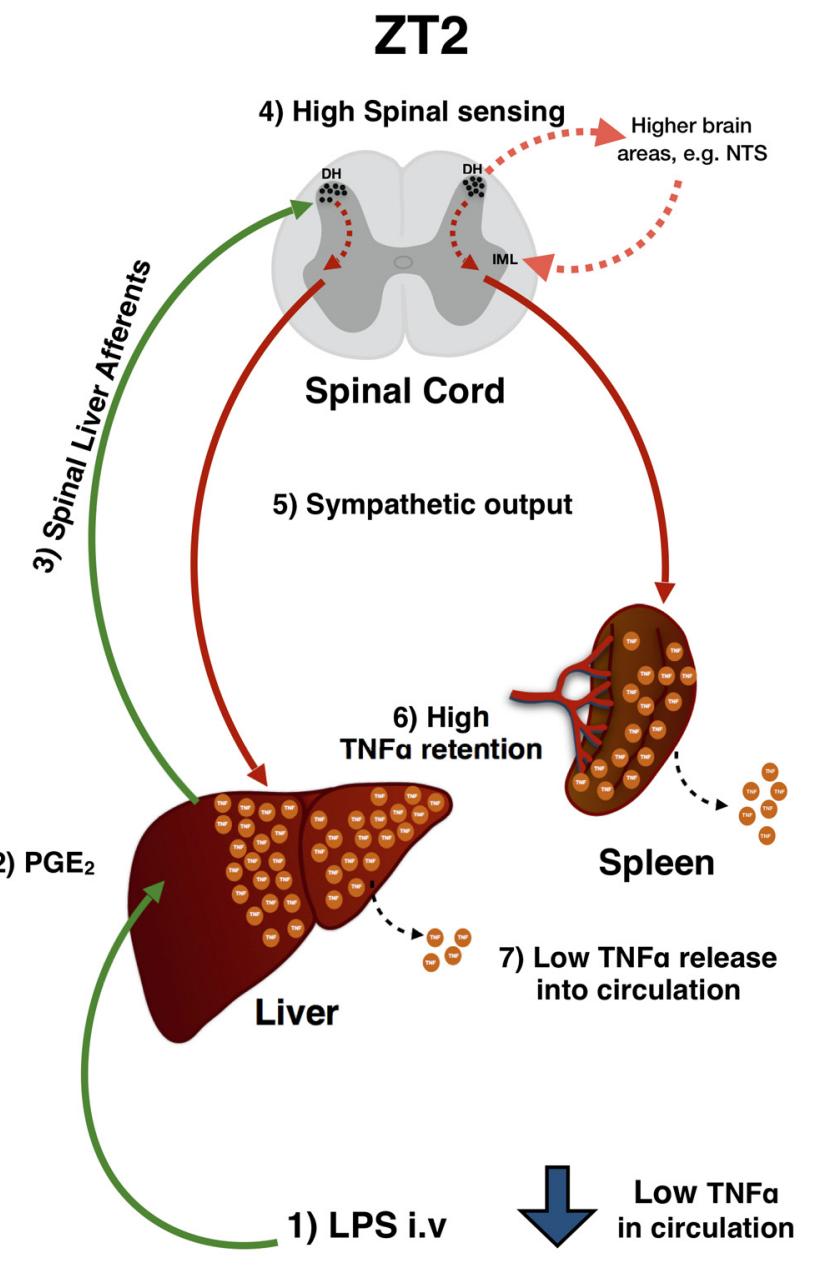

\section{ZT14}

4) Low Spinal sensing

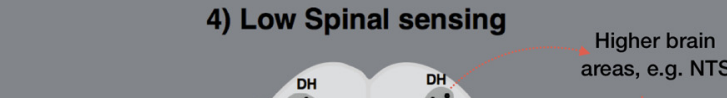

\section{0}




\section{Significance Statement}

The anti-inflammatory reflex is a survival mechanism essential for homeostasis. An out-of-control inflammatory process is detrimental to the individual and it is the cornerstone of many chronic diseases. It has been suggested that the sensory vagus nerve works as the afferent arm of this reflex arc, detecting circulating cytokines to start the anti-inflammatory reflex. The present study provides strong evidence of an additional afferent arm, which we have named the liver-spinal axis, that senses early inflammatory mediators that precede cytokine release into circulation. As an additional layer of complexity, inflammation has adapted to the light/dark cycle. We demonstrate that the anti-inflammatory reflex is time-of-day-dependent, synchronizing the peak of the inflammatory response to when it is most likely needed.

The autonomic nervous system (ANS) modulates the immune response through the engagement of an anti-inflammatory reflex. There is controversy regarding which efferent branch of the ANS, sympathetic or parasympathetic, downregulates the intensity of the inflammatory response. Furthermore, how information about the immune status of the body reaches the CNS to engage this reflex remains unclear. The present study demonstrates the existence of a liver-spinal axis that conveys early circulating inflammatory information to the CNS in response to lipopolysaccharide (LPS) and serves as the afferent arm of a sympathetic anti-inflammatory reflex. Furthermore, brainstem and spinal cord visceral sensory neurons show a time-of-day-dependent sensitivity to the incoming inflammatory information, in particular, prostaglandins (PG). Consequentially, the liverspinal axis promotes the retention of tumor necrosis factor $\alpha$ (TNF $\alpha$ ) in the liver and spleen during the resting period, resulting in low plasmatic TNF $\alpha$ levels. Consistently, low sensitivity for LPS during the active period promotes the release of TNF $\alpha$ from the organs into the circulation, resulting in high plasmatic TNF $\alpha$ levels. The present novel findings illustrate how the time-of-day-dependent activation of the liver-spinal axis contributes to the daily fluctuations of the inflammatory response.

Key words: circadian; inflammation; liver; reflex; spinal; sympathetic

\section{Introduction}

The CNS regulates the immune system through the autonomic nervous system (ANS). Through its sympathetic and parasympathetic efferent branches, the ANS is proposed to modulate the intensity of inflammatory processes

Received October 28, 2020; accepted November 9, 2020; First published November 17, 2020.

The authors declare no competing financial interests.

Author contributions: E.S.-T., E.S., and R.M.B. designed research; E.S.-T., E.S., and N.N.G.-V. performed research; M.d.C.B.-S. contributed unpublished reagents/analytic tools; E.S.-T., E.S., and R.M.B. analyzed data; E.S.-T. and R.M.B. wrote the paper.

This work was supported by the Dirección General de Asuntos del Personal Académico Grant DGAPA IG-201321 and Consejo Nacional de Ciencia y Tecnología Grants CONACyT Frontera 2017-1802 and CONACyT QUEBEC 279293 (to R.M.B.).

Acknowledgements: E.S.-T. is a PhD student from the Programa de Doctorado en Ciencias Biomédicas from the Universidad Nacional Autónoma de México and received the CONACyT Fellowship 429975. E.S. is a PhD student from the Programa de Maestría y Doctorado en Ciencias Bioquímicas from the Universidad Nacional Autónoma de México and has received the CONACyT Fellowship 695673. We thank Julio Carrero, PhD, Gloria BenitezKing, PhD and Prof. Carolina Escobar for their guidance and contributions throughout the development of this project. We also thank Dr. Martín Migliaro for the proof-reading of this manuscript. This article is dedicated to the memory of María del Carmen Basualdo Sigales (June 5th, 2020).

Correspondence should be addressed to Ruud M. Buijs at ruudbuijs@ gmail.com.

https://doi.org/10.1523/ENEURO.0463-20.2020

Copyright (C) 2020 Soto-Tinoco et al.

This is an open-access article distributed under the terms of the Creative Commons Attribution 4.0 International license, which permits unrestricted use, distribution and reproduction in any medium provided that the original work is properly attributed. through autonomic reflexes (Borovikova et al., 2000; Martelli et al., 2014). Several areas of the CNS need to be informed about the presence of a circulating inflammatory stimulus, like lipopolysaccharide (LPS), to elicit an autonomic anti-inflammatory reflex, yet, it is unclear as to how inflammatory information reaches the CNS.

One possibility is that LPS directly enters the CNS, but evidence shows that LPS has a minimal penetration across the blood-brain barrier (BBB; Banks and Robinson, 2010).

Another possibility that has been explored is that cytokines inform the CNS about the immune status of the body (Dantzer, 2001; Zanos et al., 2018). However, cytokines are detectable in circulation 20-30 min after an LPS challenge, therefore, there is a critical time-window in which other mechanisms may be essential for the early signaling of inflammatory information to the CNS. Sensory structures in the CNS expressing Toll-like receptor 4 (TLR4), an LPS receptor, are possible candidates for the immediate sensing of LPS. These include the circumventricular organs (CVOs), specialized structures located near the cerebral ventricles characterized by their lack of BBB. The expression of TLR4 was indeed reported in the four sensory CVOs: the area postrema (AP), organum vasculosum of the lamina terminalis (OVLT), subfornical organ (SFO), and median eminence (Laflamme and Rivest, 2001; Wuchert et al., 2008; Ott et al., 2010; Nakano et al., 2015), making the CVOs ideal places for detecting circulating LPS. On the other hand, TLR4 is also expressed in visceral sensory neurons located in the nodose (vagal) and dorsal root (spinal) ganglia (Hosoi et al., 
2005; Barajon et al., 2009), opening the possibility that visceral sensory neurons could detect the presence of circulating LPS. In this current study, we investigate the responsiveness of the CVOs and visceral sensory areas (VSAs) to an LPS challenge to uncover the pathways involved in the transmission of circulating inflammatory information to the CNS, as well as the ensuing response of the ANS. For the purposes of this study, we focused only on the events that take place within the first 50 min after the LPS administration. In particular, the response of tumor necrosis factor $\alpha$ (TNF $\alpha$ ), one of the first proinflammatory cytokines to be released into circulation and a major promotor and modulator of the inflammatory process (Lukacs et al., 1995; Baud and Karin, 2001). This allowed us to have a robust parameter to measure the effects of our manipulations over the inflammatory response.

Furthermore, immune processes like cytokine release (Guerrero-Vargas et al., 2014), pathogen clearance (Tognini et al., 2017), leukocyte recruitment (Scheiermann et al., 2012), and lymphocyte egress from lymph nodes (Druzd et al., 2017) are all circadian driven. In particular, the TNF $\alpha$ response to LPS is stronger during the active period than during the resting period. Previous studies have demonstrated that the ANS imposes rhythmicity on various immune processes (Méndez-Ferrer et al., 2008; Scheiermann et al., 2012), opening the question of whether the anti-inflammatory reflex contributes to the rhythmicity of the TNF $\alpha$ response to LPS.

\section{Materials and Methods}

\section{Animals and general housing conditions}

Adult male Wistar rats weighing $280-300 \mathrm{~g}$ at the beginning of the experiments were obtained from the animal facility of the Faculty of Medicine of the Universidad Nacional Autónoma de México (UNAM). Animals were group housed until they reached the desired weight for experiments. After surgery, subjects were individually housed to avoid the disruption of the implanted jugular cannula because of social interaction. The animal facility had a 12/12 h light/dark cycle with lights on at 7 A.M., defined as zeitgeber time (ZT)0, and lights off at 7 P.M., defined as ZT12. The room was maintained at a regulated temperature ranging between $22^{\circ} \mathrm{C}$ and $25^{\circ} \mathrm{C}$. All rats were given free access to water and food (Envigo Teklad 2018S). Experimental procedures used in this study were in strict accordance with the Mexican norms for animal handling (Norma Oficial Mexicana NOM-062-ZOO-1999), in agreement to international guidelines for animal handling, and approved by the Ethics Committee of the Instituto de Investigaciones Biomédicas, UNAM. All efforts were made to minimize the number of animals and their suffering.

\section{Reagents}

LPS, Escherichia coli serotype 0127:B8, (Sigma-Aldrich, catalog \#L4516, lot \#051M4004) was reconstituted with sterile water. Recombinant rat TNF $\alpha$ (Shenandoah Biolotechnology Inc., catalog \#P16599) was reconstituted with sterile water and diluted with a $0.1 \%$ BSA solution for prolonged storage. Indomethacin (Sigma-Aldrich, catalog \#17378) was reconstituted with $0.1 \mathrm{~mm} \mathrm{Na} \mathrm{CO}_{3}$. Prostaglandin $\mathrm{E}_{2}\left(\mathrm{PGE}_{2}\right.$; Cayman Chemical, catalog \#14 010) was reconstituted with $95 \%$ ethanol. Propranolol hydrochloride (Sigma-Aldrich, catalog \#P0884) was reconstituted in sterile water. All reagents were further diluted in $0.9 \%$ pyrogen-free sterile saline to yield the desired concentration for their administration to the animals.

\section{Surgical procedures}

All surgeries were performed using aseptic procedures and under deep anesthesia using a combination of intramuscular ketamine $(100 \mathrm{mg} / \mathrm{kg}$ Clorkétam 1000 Q-7090099, Vetoquinol) and xylazine $(10 \mathrm{mg} / \mathrm{kg}$, Procin, PiSA, Q-7833-099) anesthesia.

\section{Intrajugular catheter insertion}

All animals underwent the insertion of a polyethylene silicon tube catheter ( 0.025 in. i.d. and 0.047 in. o.d.; SILASTIC Laboratory tubing; Dow Corning Corp.) into the right external jugular vein. The catheter was filled with heparin $(500 \mathrm{U} / \mathrm{ml})$ as anti-coagulant and antibiotics (100 $\mathrm{U} / \mathrm{ml}$, penicillin/streptomycin, Gibco antibiotic-antimycotic $(100 \times)$, catalog \#15240062) to prevent infections. We used dental cement to secure the catheter on the top of the skull using three stainless-steel screws inserted into the skull. Through this jugular catheter, animals received the different reagents mentioned previously and also blood was withdrawn the day of the experiment. Animals were allowed to recover from this surgery for at least $7 \mathrm{~d}$ before performing the actual experiment.

\section{Telemetry transmitter implantation}

To determine whether LPS elicits a sympathetic activation, heart rate was evaluated with a Stellar telemetry transmitter (TSE Systems). The device was implanted as instructed by the manufacturer in the abdominal aorta, just rostral to the iliac bifurcation. The transmitter was sutured to the abdominal wall. Positive and negative bio-potential leads for the ECG recording were passed out of the abdomen and sutured to the left caudal rib region and to the right pectoral muscle, respectively. All incisions were closed and the animals were allowed to recover for $10 \mathrm{~d}$ before performing the actual experiment.

\section{Peritoneal capsaicin deafferentation}

To determine whether peritoneal afferent nerves play a role in the early signaling of inflammation to the CNS, we induced a peritoneal deafferentation with capsaicin. The procedure used has been described elsewhere (BretDibat et al., 1997). In brief, previously cannulated animals received a total dose of $25 \mathrm{mg} / \mathrm{kg}$ capsaicin (Natural, Sigma-Aldrich catalog \#360376) intraperitoneally, which was divided into a series of 10 injections over a 48-h period. On both days, rats were injected with ketamine (10 mg/300 g, i.v.) and xylazine (1 mg/300 g, i.v.) for anesthetic purposes and were then treated with five doses of capsaicin $(2.5 \mathrm{mg} / \mathrm{kg}$ in $300 \mu \mathrm{l}$ of vehicle (Veh): 10\% 
ethanol, $10 \%$ Tween 80 , and $80 \%$ injectable saline $0.9 \%)$. Each injection was separated from the next by a 90-min recovery period. Sham animals received the same volume of Veh as capsaicin-treated animals. Animals were allowed to recover for $12 \mathrm{~d}$ before performing the experiments.

\section{Hepatic sensory denervation \\ Spinal}

To elucidate with greater precision the involvement of hepatic sensory nerves in the early signaling of inflammation to the CNS, we performed a specific liver denervation that involved mechanical denervation followed by the application of local capsaicin to remove spinal sensory nerves. The procedure has been described elsewhere (Kalsbeek et al., 2004). On the same surgery on which the jugular cannula was placed, a laparotomy was performed in the midline. The liver lobes were gently pushed up, and ligaments around the liver lobes were severed to free the bile duct and portal vein complex, which were isolated from each other. At the level of the hepatic portal vein, the hepatic artery breaks up into the hepatic artery proper and the gastroduodenal artery. This division occurs on the ventral surface of the portal vein. At this point, the arteries were separated via blunt dissection from the portal vein. Nerve bundles running along the hepatic artery proper were removed using microsurgical instruments under an operating microscope (25× magnification). Any connective tissue attachments between the hepatic artery and portal vein were also removed, eliminating any possible nerve crossings. After this procedure, $10 \mu$ l of capsaicin $(50 \mathrm{mg} / \mathrm{ml})$ was applied on top of the bile duct and portal vein/hepatic artery complex. This procedure has been described elsewhere (Fujita et al., 2007). Spreading of capsaicin to the peritoneum was prevented by isolating this complex with a saline-soaked cotton and by using a very small volume of the capsaicin solution. The capsaicin solution was let to act and dry for $\sim 10$ min over the hepatic portal system and afterward, the peritoneum is closed. Animals were allowed to recover for $12 \mathrm{~d}$ before performing the experiments.

\section{Vagal}

To determine the role of the vagus nerve in the early signaling of inflammation to the CNS, we performed a hepatic vagotomy. Since it is known that the vagus is a mixed sensory-motor nerve, and depending on the species, $\sim 70-80 \%$ of the nerve comprises sensory fibers (Browning et al., 2017) this is a valid approach to remove the afferent vagus. The procedure has been described elsewhere (Kalsbeek et al., 2004). On the same surgery on which the jugular cannula was placed, a laparotomy was performed in the midline. The fascia containing the hepatic branch was stretched by gently moving the stomach and the esophagus. With a myelin-specific dye (Toluidin Blue), the hepatic branch could be revealed as it separates from the left vagal trunk. With the aid of a binocularoperating microscope, the stained neural tissue was transected between the ventral vagus trunk and liver. Also, small blue-stained branches running in the fascia between the stomach and the liver were transected. Afterwards, the peritoneum is closed.

As a control, we performed a sham surgery, in which a laparotomy was performed in the midline and the liver, and its nerves were manipulated, but not severed. Afterwards, the peritoneum is closed. Animals were allowed to recover for $12 \mathrm{~d}$ before performing the experiments.

\section{Sample collection and ELISA for TNF $\alpha$ and PGE P $_{2}$}

In all experiments, blood samples were collected before (time 0 was used as an inside control) and $50 \mathrm{~min}$ after the respective treatment. Hereafter, animals were deeply anesthetized with an overdose of pentobarbital iv. Afterwards, the peritoneal cavity was opened, spleen and liver were collected fresh and the animal was then perfused with saline followed by $4 \%$ paraformaldehyde in PBS. TNF $\alpha$ levels in plasma, spleen and liver, were determined with an ELISA developed in our laboratory. Rat TNF $\alpha$ mouse recombinant monoclonal antibody (R\&D Systems, catalog \#MAB510R, RRID:AB_2876875) was used as the capture antibody and Rat TNF $\alpha$ goat polyclonal biotinylated antibody (R\&D Systems, catalog \#BAF510, RRID:AB_356538) was used as the detection antibody. Following the instructions of the manufacturer, $\mathrm{PGE}_{2}$ concentration in plasma was determined with a competitive ELISA that quantifies a primary metabolite of $\mathrm{PGE}_{2}$ since the latter is too unstable to be measured directly (Cayman Chemical, catalog \#514531, RRID:AB_ 2876793).

\section{Immunohistochemistry}

Following perfusion, spinal cord, brain and brainstem were removed, postfixed for $24 \mathrm{~h}$, cryoprotected in $30 \%$ sucrose for $48-72 \mathrm{~h}$, frozen, and cut in coronal sections of $40 \mu \mathrm{m}$ at $-20^{\circ} \mathrm{C}$ in a cryostat. Free floating sections were incubated with c-Fos rabbit polyclonal antibody (1:2000; Santa Cruz Biotechnology, catalog \#sc-52) for $48 \mathrm{~h}$ at $4^{\circ} \mathrm{C}$. Later, they were rinsed and incubated with a biotinylated donkey-anti rabbit antibody (1:500, Jackson ImmunoResearch, catalog \#711-065-152, RRID: AB_2340593) and avidin-biotin complex (1:500, Vector Laboratories, catalog \#PK-4000), for $2 \mathrm{~h}$ each at room temperature. Product visualization was obtained with $0.01 \%$ diaminobenzidine, $0.05 \%$ nickel ammonium sulfate and $0.01 \%$ hydrogen peroxide for $15 \mathrm{~min}$. All sections were mounted, dehydrated, and cover-slipped with microscopy Entellan New (Merck/Sigma-Aldrich, catalog \#107961).

\section{Quantification of c-Fos immunoreactivity}

In order to quantify immunoreactivity of c-Fos in the $\mathrm{DH}$, ten representative sections of thoracical (T7-T10) level sections were selected in accordance with the Atlas of the Rat Spinal Cord (Watson et al., 2009). For the nucleus of the solitary tract (NTS) and AP, 6 representative sections were selected (bregma $-13.92,-14.16)$; for the SFO and OVLT, three representative sections were selected (bregma $-0.72,-1.08$ for the SFO and bregma 0.6, 0.36 for the OVLT), all in accordance with the Atlas of 
the Rat Brain in Stereotactic Coordinates (Paxinos and Watson, 2005). Images were examined under a light microscope and captured with a $20 \times$ ocular. An InfinityHD camera (Lumenera Software) was used to acquire all the images used in this study. Immunoreactive-positive nuclei were counted using a computerized image analysis system (ImageJ, 1.42q, National Institutes of Health) by determining the area of each nuclei by free hand. The background was subtracted and threshold was determined; particle analysis was set for particles of 1.0-2.0 circularity and $500-800$ pixels. Background optic density was established in a nearby region lacking c-Fos immunoreactivity. The average of all the counts in the different sections, was taken as the final measurement.

\section{Western blot analysis}

The procedure used has been described elsewhere (limuro et al., 1998); $5 \mathrm{mg}$ of liver tissue was weighed and lysed with $300 \mu \mathrm{l}$ of lysis buffer $(50 \mathrm{~mm}$ Tris- $\mathrm{HCl} \mathrm{pH}$ $8.0,150 \mathrm{~mm} \mathrm{NaCl}, 1 \%$ Triton $\mathrm{X}-100,0.5 \%$ sodium deoxycholate, and $1 \%$ SDS) and $50 \mu$ l of protease inhibitor stock (Roche cOmplete Protease Inhibitor Cocktail, 11697498001 , one tablet was diluted in $1 \mathrm{ml}$ of PBS). The homogenization of the tissue was performed in a TissueLyser LT (QIAGEN). Samples were centrifuged at $14,000 \mathrm{rpm}$ at $4^{\circ} \mathrm{C}$ for $10 \mathrm{~min}$, and the supernatant was collected. Protein concentration was determined with a NanoDropTM 1000 Spectrophotometer (Thermo Fisher Scientific). Forty micrograms of protein from each sample was separated in SDS-PAGE and subsequently transferred to nitrocellulose membranes. The nitrocellulose membranes were rinsed and blocked with $3 \%$ BSA in TBS for $1 \mathrm{~h}$ at room temperature and cut in half at $\sim 60 \mathrm{kDa}$. The upper half was probed with a mouse monoclonal anti-TLR4 (1:4000, Novus Biologicals, catalog \#NB100-56566, RRID: $\left.A B \_2205129\right)$, and the lower half was probed with a mouse monoclonal anti- $\alpha$-tubulin as loading control (1:5000, Sigma-Aldrich, catalog \#T5168, RRID:AB 477579 ) for $24 \mathrm{~h}$ at $4^{\circ} \mathrm{C}$. Later, both membranes were probed with a biotinylated donkey-anti mouse antibody (1:500, Jackson ImmunoResearch, catalog \#715-065150, RRID: AB_2307438) and avidin-biotin complex (1:500, Vector Laboratories, catalog \#PK-4000) for $2 \mathrm{~h}$ each at room temperature. Immunoreactivities were visualized using $0.01 \%$ diaminobenzidine, $0.05 \%$ nickel ammonium sulfate, and $0.01 \%$ hydrogen peroxide for $5 \mathrm{~min}$. Results were analyzed using ImageJ to measure the optic density of TLR4 immunoreactivity normalized with respect to the $\alpha$-tubulin control levels in the same sample.

\section{Experimental design}

During all experiments, animals were permanently connected to the blood-sampling line, which was attached to a metal collar and kept out of reach of the animals by means of a counterbalanced beam. This allowed all manipulations to be performed without handling the animals and maximally avoid a stress response. This remote manipulation also prevents the upregulation of c-Fos expression normally induced by the handling of the animals and observe only the c-Fos induced by the LPS challenge. The metal collars were attached $24 \mathrm{~h}$ before the actual experiment (Kalsbeek et al., 2012) to allow the animals to recover from the stress of the collar attachment.

\section{Experiment 1: determine which area of the CNS responds early after an LPS challenge}

Animals were cannulated and randomly assigned to one of two conditions, LPS $(2 \mu \mathrm{g} / \mathrm{kg})$ or Veh administration at ZT2 ( $n=6 /$ group). Blood samples were collected before and $50 \mathrm{~min}$ after the respective treatment for TNF $\alpha$ determination. Both groups were deeply anesthetized and transcardially perfused $50 \mathrm{~min}$ after the respective treatment. Brain, spinal cord and brainstem were processed for c-Fos immunohistochemistry.

\section{Experiment 2: determine which molecule activates the CNS after an LPS challenge}

Animals were cannulated and randomly assigned to one of five conditions: Veh $(n=6)$, LPS $(2 \mu \mathrm{g} / \mathrm{kg} ; n=6)$, $\mathrm{TNF} \alpha(5 \mu \mathrm{g} / \mathrm{kg} ; n=5)$, LPS $(2 \mu \mathrm{g} / \mathrm{kg})+$ indomethacin $(10 \mathrm{mg} / \mathrm{kg} ; n=6)$ or $\mathrm{PGE}_{2}(100 \mu \mathrm{g} / \mathrm{kg} ; n=4)$ administration at ZT2. Blood samples were collected before and 50 min after the respective treatment for TNF $\alpha$ determination. Blood samples were collected before 10 and 20 min after LPS for $\mathrm{PGE}_{2}$ determination. All groups were deeply anesthetized and transcardially perfused 50 min after the respective treatment. Spinal cord and brainstem were processed for c-Fos immunohistochemistry. Additional animals for each group (Veh, LPS, LPS + Indo, $n=3$ /group) were implanted with a telemetry transmitter to measure heart rate, which was continuously monitored at 2.5 -min intervals for $1 \mathrm{~h}$.

\section{Experiment 3: determine whether visceral sensory affer-} ents detect LPS and start an anti-inflammatory reflex

Animals were cannulated and randomly assigned to one of two conditions: peritoneal deafferentation with capsaicin or sham ( $n=5 /$ group). Animals were allowed to recover for $12 \mathrm{~d}$. Other series of animals were cannulated and randomly assigned to one of three conditions: sham, vagal sensory liver denervation (VLX), or spinal sensory liver denervation (SLX; $n=6$ /group). Animals were allowed to recover for $12 \mathrm{~d}$. The day of the experiment, all animals were challenged with LPS $(2 \mu \mathrm{g} / \mathrm{kg})$ at ZT2. An additional set of animals received LPS or LPS + propranolol $(1 \mathrm{mg} /$ $\mathrm{kg}$ ) at ZT2 ( $n=5 /$ group). Blood samples were collected before and $50 \mathrm{~min}$ after the respective treatment for TNF $\alpha$ determination. All animals were deeply anesthetized and transcardially perfused $50 \mathrm{~min}$ after their respective treatment. Spinal cord and brainstem were processed for c-Fos immunohistochemistry. Liver and spleen were processed for TNF $\alpha$ determination.

Experiment 4: determine whether the plasma TNF $\alpha$ rhythm is because of a time-of-day-dependent sensitivity to LPS in the VSAs

Animals were cannulated and randomly assigned to one of two conditions: LPS administration at ZT2 or LPS 
Table 1: Statistical table

\begin{tabular}{|c|c|c|c|c|}
\hline Figure & Panel & Distribution & Statistical test (frequentist) & Statistical value and $p$ value \\
\hline \multirow[t]{3}{*}{1} & $D$ & Normal distribution & Unpaired $t$ test & $t=0.5000 \mathrm{df}=4 p=0.6433(\mathrm{Cl}=95 \%)$ \\
\hline & $F$ & Normal distribution & Unpaired $t$ test & $t=0.7559 \mathrm{df}=4 p=0.4918(\mathrm{Cl}=95 \%)$ \\
\hline & $H$ & Normal distribution & Unpaired $t$ test & $t=0.7071 \mathrm{df}=4 p=0.5185(\mathrm{Cl}=95 \%)$ \\
\hline \multirow[t]{5}{*}{2} & C & Normal distribution & One-way ANOVA + Tukey's post hoc test & $F_{(2,6)}=81.85 p<0.0001(\mathrm{Cl}=95 \%)$ \\
\hline & $E$ & Normal distribution & One-way ANOVA + Tukey's post hoc test & $F_{(2,6)}=79.53 p<0.0001(\mathrm{Cl}=95 \%)$ \\
\hline & $G$ & Normal distribution & $\begin{array}{l}\text { One-way RM ANOVA + Dunnett's post } \\
\text { hoc test }\end{array}$ & $F_{(1.306,9.142)}=106.2 p<0.0001(\mathrm{Cl}=95 \%$ \\
\hline & $H$ & Normal distribution & $\begin{array}{l}\text { One-way RM ANOVA + Dunnett's post } \\
\text { hoc test }\end{array}$ & $F_{(1.001,2.003)}=31.03 p=0.0306(\mathrm{Cl}=95 \%)$ \\
\hline & I & Normal distribution & $\begin{array}{l}\text { One-way RM ANOVA + Dunnett's post } \\
\text { hoc test }\end{array}$ & $F_{(1.254,2.508)}=74.99 p=0.0061(\mathrm{Cl}=95 \%)$ \\
\hline \multirow[t]{2}{*}{3} & $A$ & Normal distribution & $\begin{array}{l}\text { Two-way RM ANOVA + Sidak's post hoc } \\
\text { test }\end{array}$ & $F_{(2,8)}=13.63 p=0.0026(\mathrm{Cl}=95 \%)$ \\
\hline & C & Normal distribution & One-way ANOVA + Tukey's post hoc test & $F_{(2,6)}=30.98 p=0.0007(\mathrm{Cl}=95 \%)$ \\
\hline
\end{tabular}

E

Normal distribution One-way ANOVA + Tukey's post hoc test $F_{(26)}=58.42 p=0.0001(\mathrm{Cl}=95 \%)$

Normal distribution One-way ANOVA + Tukey's post hoc test $\quad F_{(3,20)}=33.97 p<0.0001(\mathrm{Cl}=95 \%)$

C

Normal distribution Two-way RM ANOVA + Tukey's post hoc test

D Normal distribution Unpaired $t$ test

5 Cormal distribution Unpaired $t$ test

E Normal distribution Unpaired $t$ test

G Normal distribution Unpaired $t$ test

$t=3.658 \mathrm{df}=9 p=0.0052(\mathrm{Cl}=95 \%)$

6

Normal distribution One-way ANOVA + Tukey's post hoc test

$F_{(2,15)}=60.39 p<0.0001(\mathrm{Cl}=95 \%)$

E

Normal distribution One-way ANOVA + Tukey's post hoc test $\quad F_{(2,15)}=275.2 p<0.0001(\mathrm{Cl}=95 \%)$

Panel Effect size and $\mathrm{Cl}$ (estimation statistics)

$0.0169[95.0 \% \mathrm{Cl}-1.2,1.2] p=0.97$

$0.0209[95.0 \% \mathrm{Cl}-1.19,1.26] p=0.841$

$0.0758[95.0 \% \mathrm{Cl}-1.21,1.29] p=0.886$ VEH vs LPS $=9.47[95.0 \% \mathrm{Cl} 6.63,12.4] p=0.0$ $\mathrm{VEH}$ vs TNF $\alpha=-0.0633[95.0 \% \mathrm{Cl}-1.22,1.24]$

$$
p=0.908
$$

VEH vs LPS $=8.55[95.0 \% \mathrm{Cl} 6.44,11.1] p=0.0$ VEH vs TNF $\alpha=0.563[95.0 \% \mathrm{Cl}-0.756,1.59] p=$ 0.331

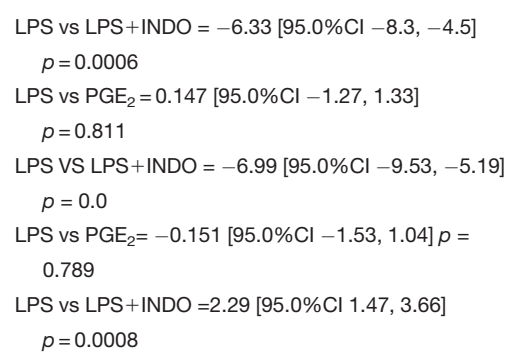

$$
t=12.74 \mathrm{df}=8 p<0.0001(\mathrm{Cl}=95 \%)
$$

$D$

F

$H$

$F$

$B$

$D$

(Continued)
LPS vs LPS + Pro $=1.66[95.0 \% \mathrm{Cl} 0.575,2.68] p=$ 0.0162

SHAM+LPS vs CAPS + LPS $=-8.11[95.0 \% \mathrm{Cl}$

$-10.2,-6.04] p=0.0088$

SHAM+LPS vs CAPS + LPS $=-9.28[95.0 \% \mathrm{Cl}$

$-14.4,-6.8] p=0.0062$

SHAM+LPS vs CAPS + LPS $=2.49[95.0 \% \mathrm{Cl} 1.67$, 7.37] $p=0.003$.

SHAM+LPS vs VLX+LPS $=-1.6[95.0 \% \mathrm{Cl}-3.37$, $-0.235] p=0.0136$

SHAM+LPS vs SLX+LPS $=-8.12[95.0 \% \mathrm{Cl}-11.8$, $-5.38] p=0.0$

SHAM+LPS vs VLX+LPS $=-8.99[95.0 \% \mathrm{Cl}-13.0$, $-6.12] p=0.0$

SHAM+LPS vs SLX+LPS $=-12.4[95.0 \% \mathrm{Cl}-21.8$, $-7.79] p=0.0$

SHAM + LPS vs VLX +LPS $=-0.879[95.0 \% \mathrm{Cl}$ $-2.49,0.604] p=0.12$

SHAM+LPS vs SLX+LPS $=3.86[95.0 \% \mathrm{Cl} 2.51$, 5.21] $p=0.0$

SHAM + LPS vs VLX +LPS $=-0.187[95.0 \% \mathrm{Cl}$

$-1.65,1.3] p=0.742$

SHAM+LPS vs SLX+LPS $=29.0[95.0 \% \mathrm{Cl} 18.7$, 59.1] $p=0.0$

SHAM+LPS vs VLX+LPS $=-0.962[95.0 \% \mathrm{Cl}$

$-1.96,0.218] p=0.0972$

SHAM + LPS vs SLX+LPS $=5.07[95.0 \% \mathrm{Cl} 3.81$, 17.3] $p=0.0$

LPS ZT2 vs LPS ZT14 $=-7.04[95.0 \% \mathrm{Cl}-8.91$, $-5.26] p=0.0066$

LPS ZT2 vs LPS ZT14 $=-7.27[95.0 \% \mathrm{Cl}-9.42$, $-5.91] p=0.005$ 
Table 1: Continued

\begin{tabular}{|c|c|c|c|c|c|c|}
\hline Figure & Panel & Distribution & Statistical test (frequentist) & Statistical value and $p$ value & Panel & Effect size and $\mathrm{Cl}$ (estimation statistics) \\
\hline & $G$ & Normal distribution & Unpaired $t$ test & $t=5.772 \mathrm{df}=15 p<0.0001(\mathrm{Cl}=95 \%)$ & $H$ & LPS ZT2 vs LPS ZT14 = $8.32[95.0 \% \mathrm{Cl} 6.24,10.8]$ \\
\hline & & & & & & $p=0.0008$ \\
\hline & I & Normal distribution & $\begin{array}{l}\text { Two-way RM ANOVA + Sidak's post hoc } \\
\text { test }\end{array}$ & $F_{(2,8)}=1.070 p=0.3873(\mathrm{Cl}=95 \%)$ & & \\
\hline \multirow[t]{8}{*}{9} & C & Normal distribution & Unpaired $t$ test & $t=6.067 \mathrm{df}=4 p=0.0037(\mathrm{Cl}=95 \%)$ & $D$ & Low LPS ZT14 vs High LPS ZT14 = $9.94[95.0 \% \mathrm{Cl}$ \\
\hline & & & & & & $7.51,12.6] p=0.0042$ \\
\hline & E & Normal distribution & Unpaired $t$ test & $t=15.62 \mathrm{df}=4 p<0.0001(\mathrm{Cl}=95 \%)$ & $F$ & Low LPS ZT14 vs High LPS ZT14=10.7 $[95.0 \% \mathrm{Cl}$ \\
\hline & & & & & & $8.23,13.2] p=0.0$ \\
\hline & G & Normal distribution & Unpaired $t$ test & $t=0.1512 \mathrm{df}=4 p=0.8871(\mathrm{Cl}=95 \%)$ & $H$ & $\mathrm{ZT} 2$ vs $\mathrm{ZT} 14=-0.0985[95.0 \% \mathrm{Cl}-5.42,1.57] p=$ \\
\hline & & & & & & 0.809 \\
\hline & $J$ & Normal distribution & Unpaired $t$ test & $t=10.27 \mathrm{df}=6 p<0.0001(\mathrm{Cl}=95 \%)$ & $J$ & LPS CT2 vs LPS CT14 = $6.64[95.0 \% \mathrm{Cl} 5.01,9.7]$ \\
\hline & & & & & & $p=0.0016$ \\
\hline \multirow[t]{6}{*}{10} & $A$ & Normal distribution & Unpaired $t$ test & $t=6.140 \mathrm{df}=9 p<0.0002(\mathrm{Cl}=95 \%)$ & $B$ & LPS ZT2 vs LPS ZT14 = -3.59 [95.0\% Cl -6.24 , \\
\hline & & & & & & $-2.13] p=0.0008$ \\
\hline & C & Normal distribution & Unpaired $t$ test & $t=4.135 \mathrm{df}=10 p<0.002(\mathrm{Cl}=95 \%)$ & $D$ & LPS ZT2 vs LPS ZT14 = -2.2 [95.0\%Cl -3.76 , \\
\hline & & & & & & $-1.26] p=0.0$ \\
\hline & E & Normal distribution & One-way ANOVA + Tukey's post hoc test & $F_{(3,12)}=8.659 p=0.0025(\mathrm{Cl}=95 \%)$ & $F$ & LPS vs LPS +INDO $=-0.352[95.0 \% \mathrm{Cl}-1.89,0.851]$ \\
\hline & & & & & & $p=0.529$ \\
\hline
\end{tabular}

One-way or two-way ANOVA, repeated measures (RM), confidence intervals (CI).

administration at ZT14 ( $2 \mu \mathrm{g} / \mathrm{kg} ; n=5 / \mathrm{group})$. Another set of animals ( $n=5 /$ group) was kept in constant darkness from the night before the experiment started. In these experiments, time is indicated as circadian time (CT), indicating that the animal's endogenous rhythm of $\sim 24 \mathrm{~h}$ is not entrained to external cues, in this case, light. These animals received LPS $(2 \mu \mathrm{g} / \mathrm{kg})$ at CT2 $(2 \mathrm{~h}$ after the beginning of the subjective day) or CT14 (2 h after the beginning of the subjective night). Another set of animals was randomly assigned to one of three conditions: Veh, LPS, or LPS +indomethacin $(10 \mathrm{mg} / \mathrm{kg})$ administration at ZT14 ( $n=5-6 /$ group). Another set of animals was randomly assigned to one of two conditions: low LPS $(2 \mu \mathrm{g} /$ $\mathrm{kg})$ or high LPS $(100 \mu \mathrm{g} / \mathrm{kg})$ administration at ZT14 ( $n=5 /$ group). Blood samples were collected before and 50 min after the respective treatment for TNF $\alpha$ determination. Blood samples were collected before 10 and 20 min after LPS for $\mathrm{PGE}_{2}$ determination. All animals were deeply anesthetized and transcardially perfused 50 min after their respective treatment. Spinal cord and brainstem were processed for c-Fos immunohistochemistry. Liver and spleen were processed for TNF $\alpha$ determination. An additional set of animals ( $n=3 /$ group) was killed without any treatment at ZT2 or ZT14 to evaluate the basal TLR4 expression in the liver by Western blotting.

\section{Data analysis and statistics}

Animals were randomly assigned to experimental time points or groups. Investigators were blinded to group allocation during data analysis. Animals that showed detectable levels of TNF $\alpha$ before the administration of LPS were excluded from the final analysis. Data analysis was performed using GraphPad Prism 6. All data were normally distributed and are expressed as mean \pm SEM.

For the present study, we analyzed the data with two types of statistics (frequentist and estimation statistics) to evaluate significance as well as quantitatively express size effects and its precision. In bold bars or continuous curves, we show the data analyzed with frequentist statistics. The statistical tests, exact $n$ values, $p$ values, and degrees of freedom are described in detail in Table 1, in each figure and figure legend. In brief, a Student's $t$ test was used when a comparison between two independent groups was needed. For multiple groups, data were analyzed using a one-way ANOVA followed by a Tukey's post hoc test. Data from several time points were analyzed using a one-way ANOVA for repeated measures followed by a Dunnett's post hoc test; or a two-way ANOVA for repeated measures, followed by a Sidak's or Tukey's post hoc test; $p<0.05$ was considered statistically significant.

For the estimation statistics, we introduced the raw data in https://www.estimationstats.com/ and downloaded the results and graphs (Ho et al., 2019). We did not use the estimation statistics for data that were originally analyzed with one-way or two-way ANOVA for repeated measures.

To measure the effect size, we used the Hedges' $g$ estimate, which is the mean difference between two groups divided by the pooled standard deviation of both groups, corrected for small samples $(<20)$. The effect sizes and confidence intervals (Cls) are reported in detail in Table 1 and in each figure as: effect size $(\mathrm{Cl}$ width lower bound; upper bound). As a rule of thumb for the interpretation of the Hedges' $g$, an effect size $\leq 0.2$ describes a small effect; an effect size $\leq 0.5$ describes a medium effect and an effect size $\leq 0.8$ describes a large effect.

When comparing two groups, the Hedges' $g$ between control and test is shown with a Gardner-Altman estimation plot. The raw data from both groups are plotted on the left axes; the mean difference is plotted on a floating axis on the right as a bootstrap sampling distribution. The mean difference is depicted as a dot; the $95 \% \mathrm{Cl}$ is indicated by the ends of the vertical error bar. 

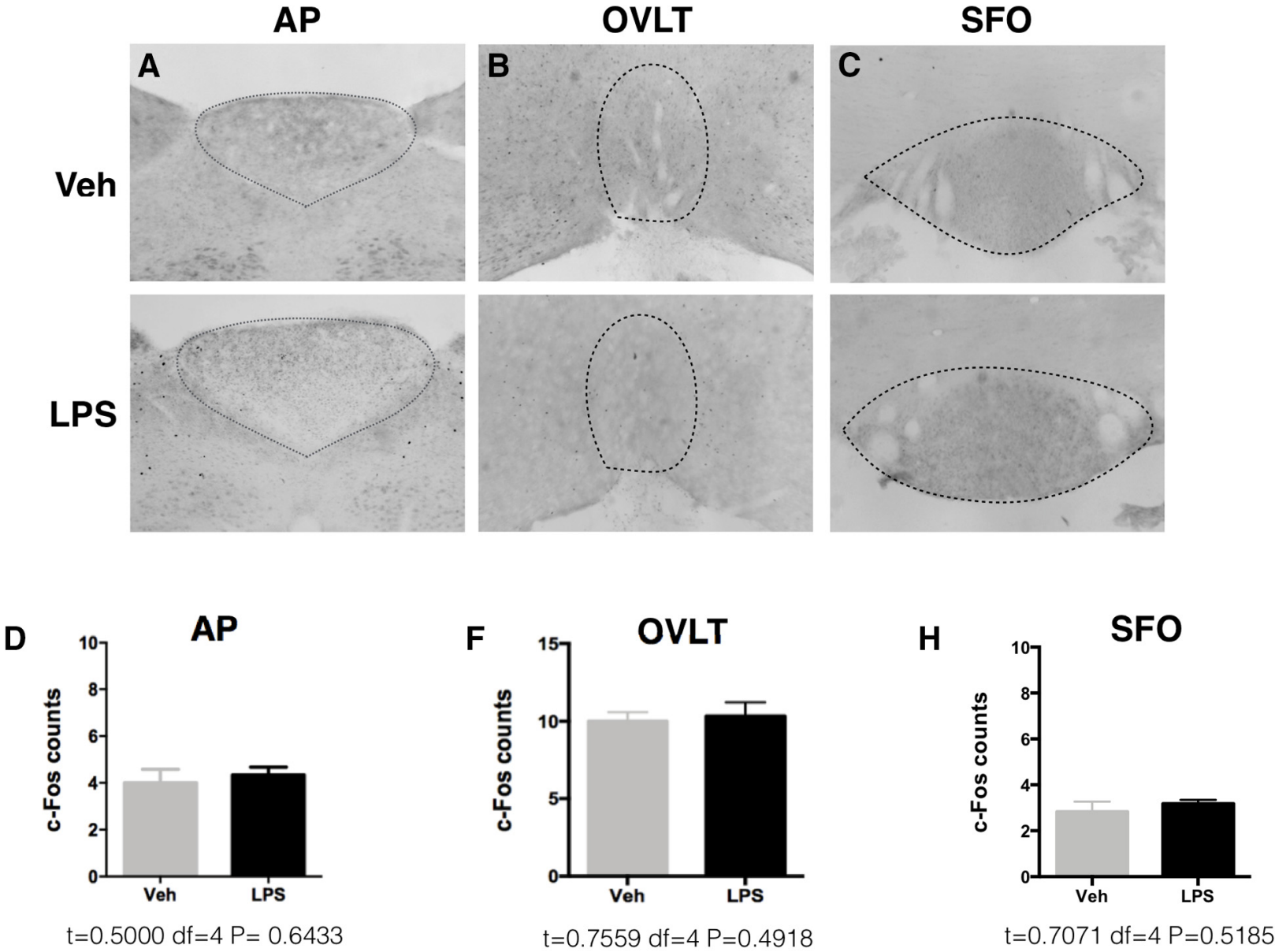

E

G

I

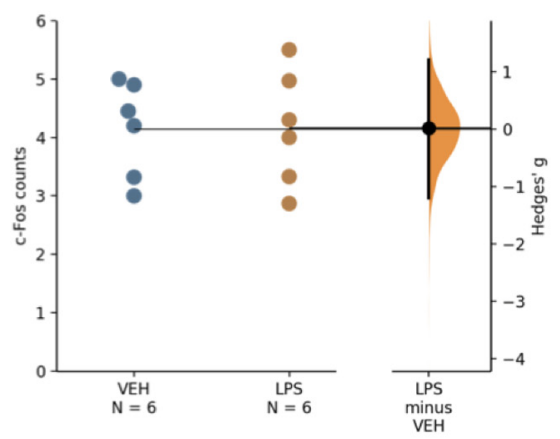

VEH vs LPS $=0.0169[95.0 \% \mathrm{Cl}$

$$
-1.2,1.2] P=0.97
$$

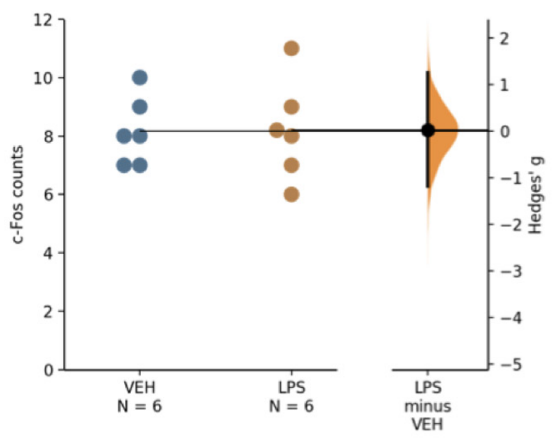

VEH vs LPS $=0.0209[95.0 \% \mathrm{Cl}$ $-1.19,1.26] \mathrm{P}=0.841$

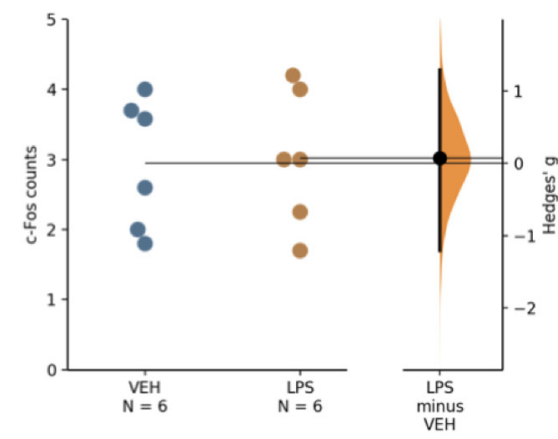

VEH vs LPS $=0.0758[95.0 \% \mathrm{Cl}$ $-1.21,1.29] \mathrm{P}=0.886$

Figure 1. Circulating LPS does not activate the CVOs. Representative pictures showing c-Fos expression in the CVOs: AP $(\boldsymbol{A})$, OVLT $(\boldsymbol{B})$, SFO $(\boldsymbol{C}) 50 \mathrm{~min}$ after Veh or LPS $(2 \mu \mathrm{g} / \mathrm{kg})$ intravenous administration at ZT2. $\boldsymbol{D}, \boldsymbol{F}, \boldsymbol{H}$, Quantification of c-Fos immunoreactivity for each structure ( $n=6$ /group). Data were analyzed with an unpaired two-tailed $t$ test. $\boldsymbol{E}$, G, $\boldsymbol{I}$, GardnerAltman estimation plot displaying the raw data from $\boldsymbol{D}, \boldsymbol{F}, \boldsymbol{H}$, the effect size and the $95 \% \mathrm{Cl}$.

When comparing three groups, the Hedges' $g$ for two comparisons against a shared control are shown with a Cumming estimation plot. The raw data are plotted on the upper axes. On the lower axes, mean differences are plotted as bootstrap sampling distributions. Each mean difference is depicted as a dot.
Each $95 \% \mathrm{Cl}$ is indicated by the ends of the vertical error bars. For each group, summary measurements (mean \pm SD) are shown as gapped lines.

In all cases, 5000 bootstrap samples were taken; the $\mathrm{Cl}$ is bias-corrected and accelerated. The $p$ value(s) reported are the likelihood(s) of observing the effect size 
DH
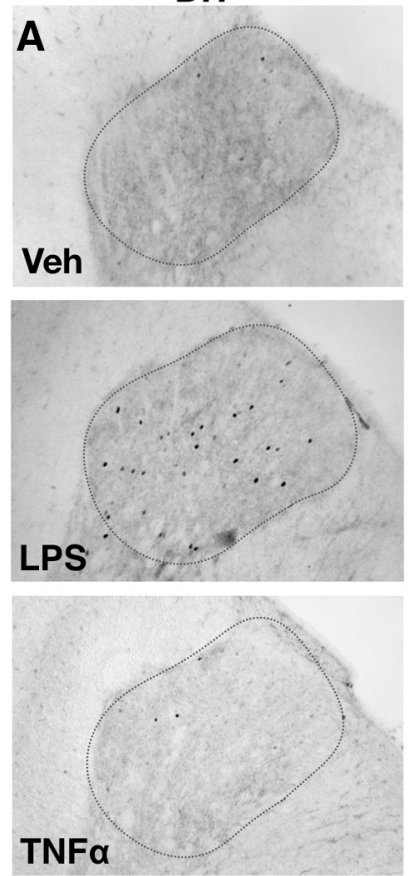

TNFa
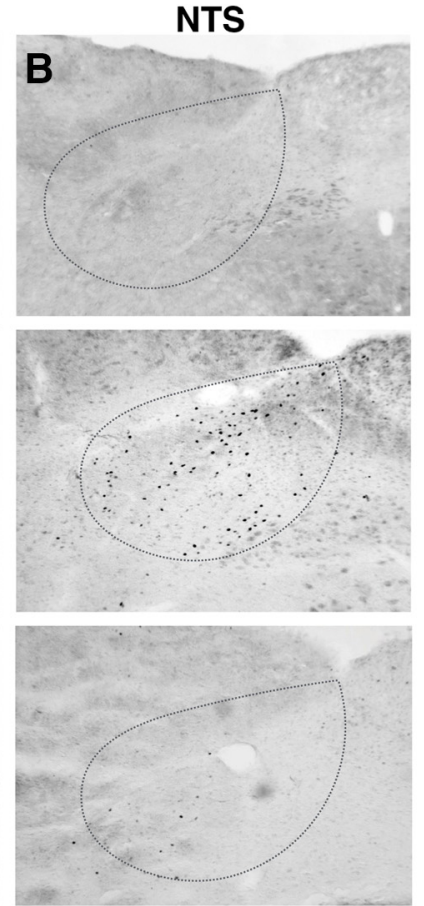

C

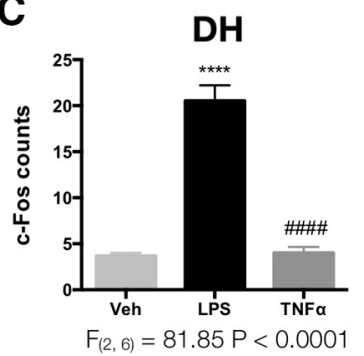

D
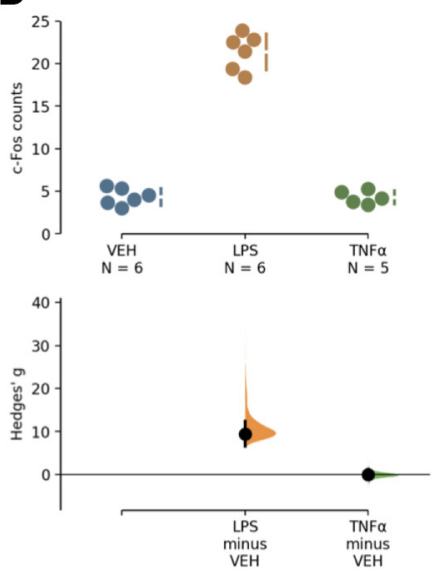

VEH vs LPS $=9.47[95.0 \% \mathrm{Cl}$ $6.63,12.4] P=0.0$ VEH vs TNFa $=-0.0633[95.0 \% \mathrm{Cl}$ $-1.22,1.24] \mathrm{P}=0.908$

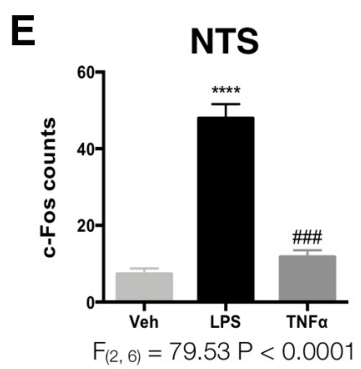

$\mathbf{F}$
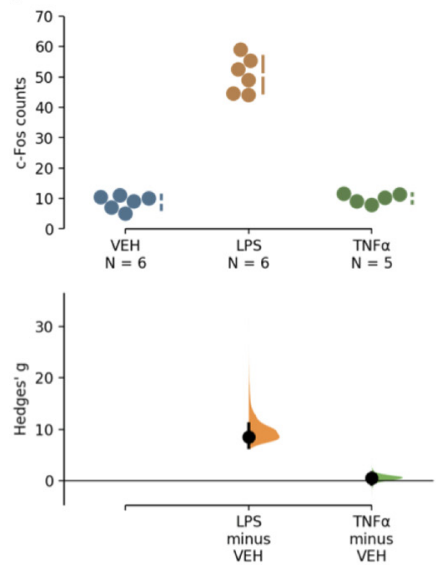

VEH vs LPS $=8.55[95.0 \% \mathrm{Cl}$ $6.44,11.1] P=0.0$ VEH vs $\mathrm{TNFa}=0.563[95.0 \% \mathrm{Cl}$ $-0.756,1.59] P=0.331$
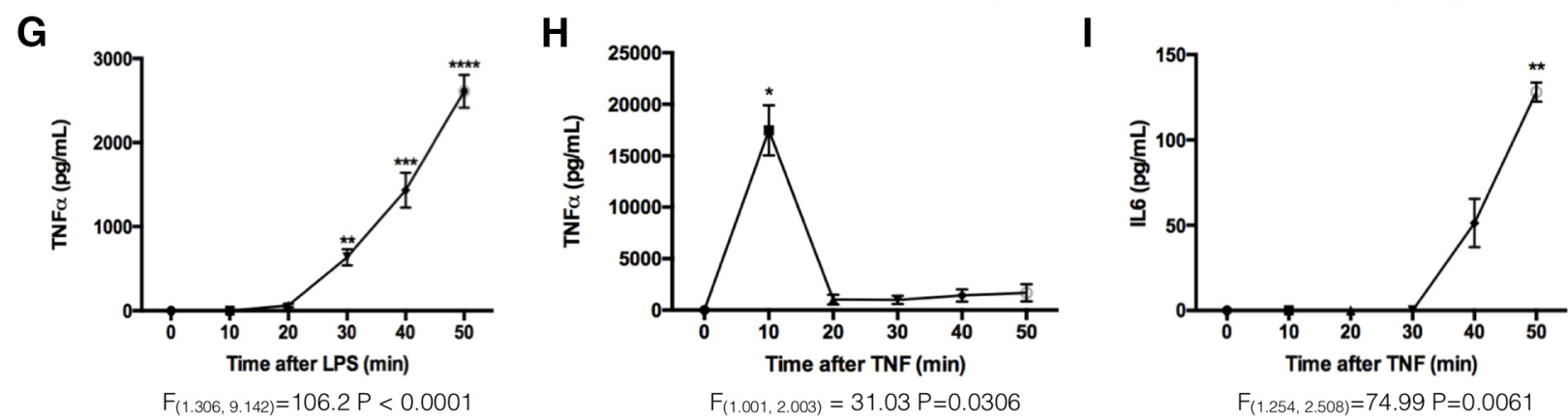

Figure 2. LPS and not TNF $\alpha$ induces neuronal activation in the VSAs. Representative pictures showing c-Fos expression in the VSAs: DH of the spinal cord $(\boldsymbol{A})$ and NTS $(\boldsymbol{B}) 50 \mathrm{~min}$ after Veh, LPS $(2 \mu \mathrm{g} / \mathrm{kg})$, or TNF $\alpha(5 \mu \mathrm{g} / \mathrm{kg})$ intravenous administration at ZT2. $\boldsymbol{C}, \boldsymbol{E}$, Quantification of c-Fos immunoreactivity for each structure ( $\mathrm{n}=5-6 / \mathrm{group})$. Data were analyzed with a one-way ANOVA fol-

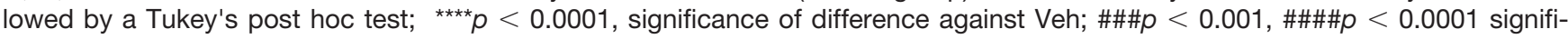
cance of difference against LPS. $\boldsymbol{D}, \boldsymbol{F}$, Cumming estimation plot displaying the raw data from $\boldsymbol{C}, \boldsymbol{E}$, the effect size and the $95 \% \mathrm{Cl}$. $\boldsymbol{G}$, Time course of TNF $\alpha$ levels in plasma after the intravenous administration of LPS at ZT2. $\boldsymbol{H}$, Time course of TNF $\alpha$ concentration in plasma after intravenous administration of TNF $\alpha$ at ZT2. I, Time course of IL-6 concentration in plasma after intravenous administration of TNF $\alpha$ at ZT2. Data in $\boldsymbol{G}, \boldsymbol{H}, \boldsymbol{I}(n=5-6 /$ group) were analyzed with a one-way ANOVA for repeated measures followed by Dunnett's post hoc test; ${ }^{*} p<0.05$, ${ }^{\star *} p<0.01$, ${ }^{* \star *} p<0.001$, ${ }^{\star * \star *} p<0.0001$ significance of difference against time 0 .

(s), if the null hypothesis of zero difference is true. For each permutation $p$ value, 5000 reshuffles of the control and test labels were performed.

\section{Results}

\section{LPS administration activates VSAs and not the CVOs}

Male Wistar rats received LPS $(2 \mu \mathrm{g} / \mathrm{kg})$ or Veh intravenously at ZT2 ( $2 \mathrm{~h}$ after the onset of the resting period) and were killed 50 min later, at almost ZT3. We found that under these experimental conditions, LPS administration did not induce c-Fos expression in any of the sensory CVOs previously suggested to participate in LPS sensing (Laflamme and Rivest, 2001; Nakano et al., 2015), indicating that these areas are not able to sense LPS in an early phase (Fig. 1).

In the same animals, we evaluated the c-Fos expression in the VSAs (Fig. 2). The dorsal horn (DH) of the spinal 


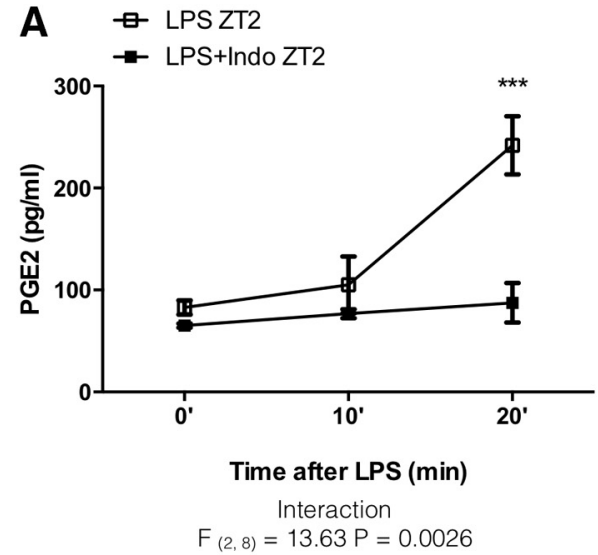

LPS+INDO
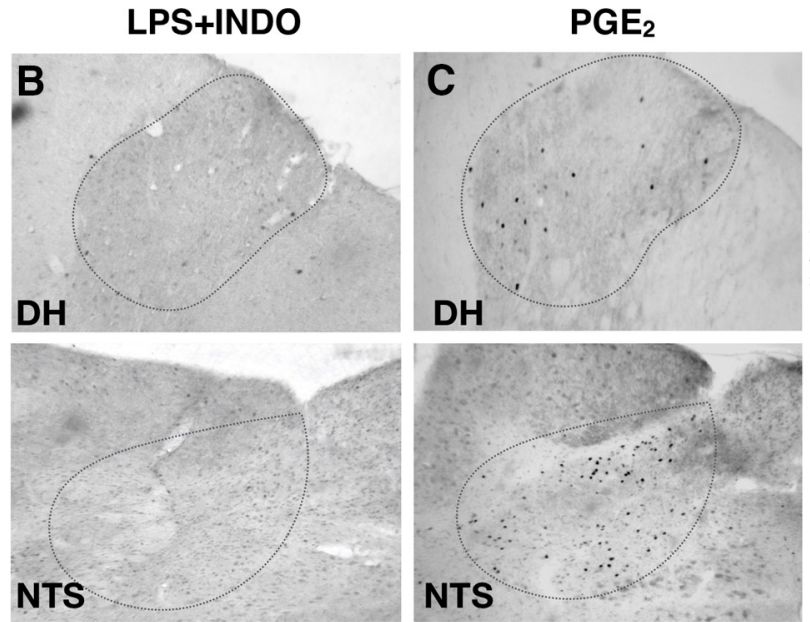

D

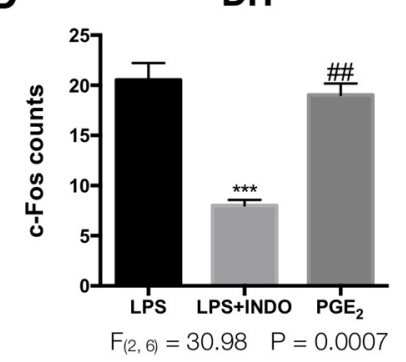

E

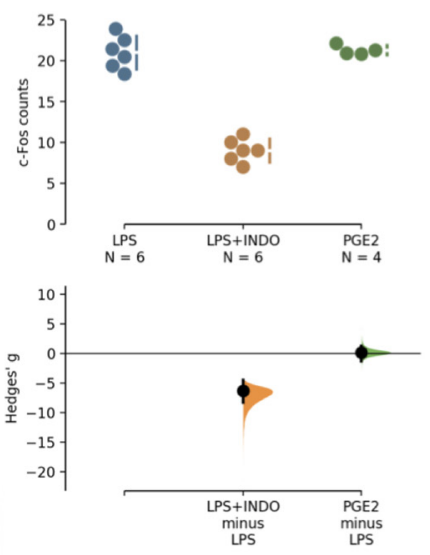

LPS vs LPS+INDO $=-6.33[95.0 \% \mathrm{Cl}$ $-8.3,-4.5] \mathrm{P}=0.0006$

LPS vs PGE2 $=0.147[95.0 \% \mathrm{Cl}-1.27$, 1.33] $\mathrm{P}=0.811$
$\mathbf{F}$

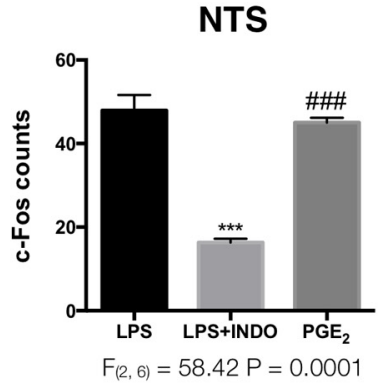

G
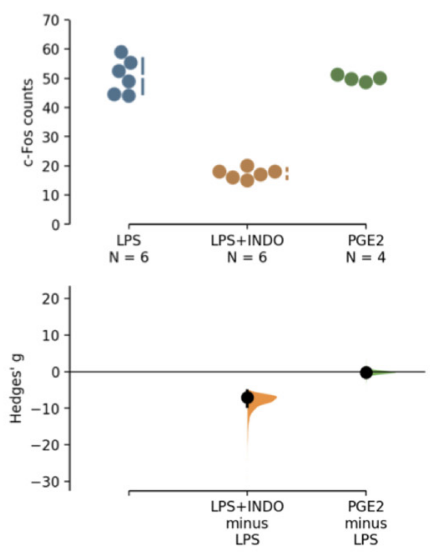

LPS VS LPS+INDO= $-6.99[95.0 \% \mathrm{Cl}$ $-9.53,-5.19] P=0.0$

LPS vs PGE2 $=-0.151[95.0 \% \mathrm{Cl}-1.53$, 1.04] $P=0.789$

Figure 3. PGs are important mediators for the LPS-induced neuronal activation in the VSAs. $\boldsymbol{A}$, PGE 2 levels in plasma at different times after LPS or LPS+Indomethacin (Indo; $10 \mathrm{mg} / \mathrm{kg}$ ) administration ( $n=6 / \mathrm{group}$ ). Data were analyzed with a two-way ANOVA for repeated measures followed by a Sidak's post hoc test; ${ }^{\star \star *} p<0.001$ significance of difference between LPS and LPS+Indo. Representative pictures showing c-Fos expression in DH and NTS 50 min after $(\boldsymbol{B})$ LPS+lndo or $(\boldsymbol{C})$ PGE $_{2}(100 \mu \mathrm{g} / \mathrm{kg})$ intravenous administration at ZT2. $\boldsymbol{D}, \boldsymbol{F}$, Quantification of c-Fos immunoreactivity for each structure ( $n=4-6$ group). Data were analyzed with a one-way ANOVA followed by a Tukey's post hoc test; ${ }^{* \star *} p<0.001$, significance of difference against LPS; \#\#\#p <0.001, \#\#p < 0.01 significance of difference against LPS+Indo. $\boldsymbol{E}, \boldsymbol{G}$, Cumming estimation plot displaying the raw data from $\boldsymbol{D}, \boldsymbol{F}$, the effect size and the $95 \% \mathrm{Cl}$.

cord exclusively receives spinal sensory information, while the NTS located in the brainstem receives both vagal and spinal sensory input. We found that c-Fos expression in these areas was significantly increased after the LPS challenge [DH (Fig. 2C,D) NTS (Fig. 2E,F)], suggesting that the VSAs do play a role in the early detection of circulating LPS.

\section{Prostaglandins (PG) are important mediators for the LPS-induced neuronal activation in the VSAs}

As aforementioned, it has been proposed that TNF $\alpha$ can directly signal the inflammatory status of the body to the CNS (Hansen et al., 2001; Dantzer et al., 2008; Zanos et al., 2018). However, detectable traces of TNF $\alpha$ in circulation only appear 20-30 min after LPS administration (Fig. 2G). Since c-Fos protein requires 40-60 min to be expressed (Sharp et al., 1991; Hoffman et al., 1993; Lin et al., 2011; Zhong et al., 2014), any c-Fos expression because of TNF $\alpha$ would only be observable after a survival time of $80 \mathrm{~min}$. Considering that we used a survival time of $50 \mathrm{~min}$, neuronal activation cannot be credited to peripheral $\operatorname{TNF} \alpha$. To further rule out the participation of TNF $\alpha$ in the activation of VSAs, we administered recombinant TNF $\alpha(5 \mu \mathrm{g} / \mathrm{kg}$, i.v. $)$ at ZT2. This dose of TNF $\alpha$ was enough to reach a concentration of $\sim 17 \mathrm{ng} / \mathrm{ml}$ in plasma (Fig. 2H) and induce the secretion of IL-6 (Fig. 2I), proving that this TNF $\alpha$ administration can effectively activate the immune response. Although the concentrations of exogenous TNF $\alpha$ exceeded the LPS-induced TNF $\alpha$ concentrations, this cytokine alone could not induce any neuronal activation in the VSAs within $50 \mathrm{~min}$ [DH (Fig. 2C,D); NTS (Fig. 2E,F)]. Therefore, we can discard the possibility that TNF $\alpha$ contributes to the early LPS-induced neuronal activation.

PGs play an important role in inflammatory networks and are released 15-20 min after LPS (Li et al., 2006; Fig. $3 A)$, which led us to suspect that they could be among the earliest molecules sensed by the VSAs. Blocking PG 


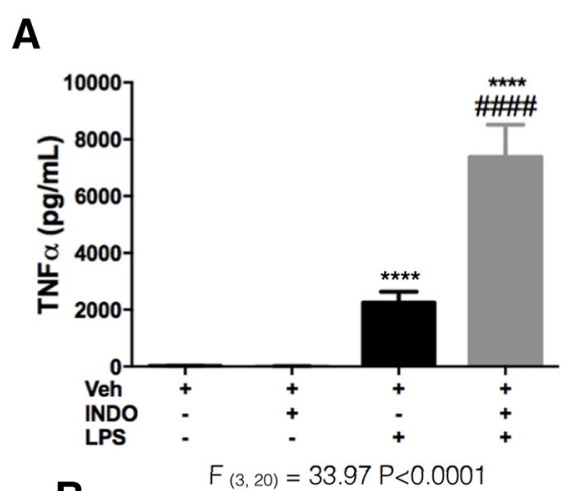

B

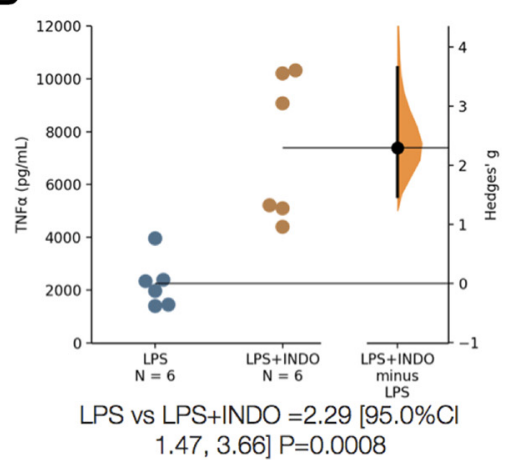

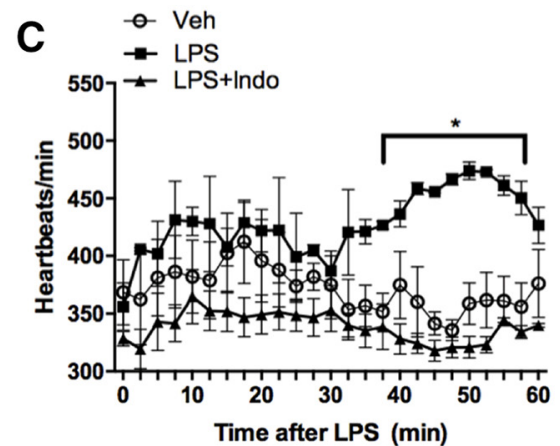

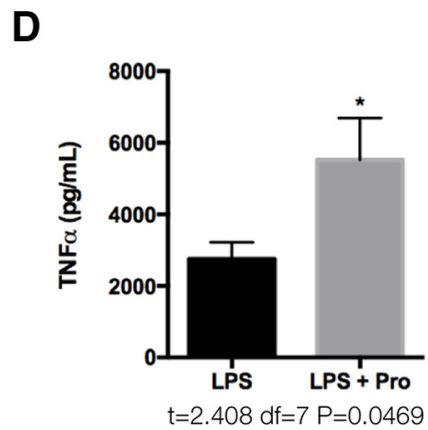

Interaction

$F_{(48,144)}=1.73 P=0.0067$
E

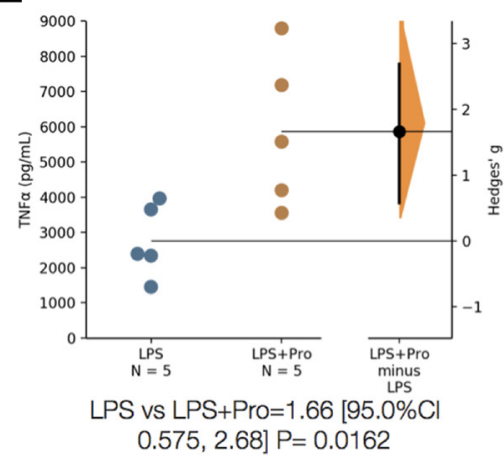

Figure 4. Detection of PG starts an early sympathetic anti-inflammatory reflex. $\boldsymbol{A}, \mathrm{TNF} \alpha$ levels in plasma 50 min after treatment with LPS or LPS+Indo ( $n=6$ /group). Data were analyzed with a one-way ANOVA followed by a Tukey's post hoc test; ${ }^{* \star * *} p<0.0001$, significance of difference against Veh; \#\#\#\#p $<0.0001$ significance of difference against LPS. B, Gardner-Altman estimation plot displaying the raw data from A, the effect size and the $95 \% \mathrm{Cl}$. C, Heart rate measurement during 60 min after Veh, LPS, or LPS + Indo administration ( $n=3$ /group). Data were analyzed with a two-way ANOVA for repeated measures followed by a Tukey's post hoc test; ${ }^{*} p<0.05$ significance of difference against LPS. $\boldsymbol{D}$, TNF $\alpha$ levels in plasma after treatment with LPS or LPS + propranolol (Pro; $1 \mathrm{mg} / \mathrm{kg} ; n=5 /$ group). Data were analyzed with an unpaired two-tailed $t$ test; ${ }^{*} p<0.05$ significance of difference against LPS. $\boldsymbol{E}$, Gardner-Altman estimation plot displaying the raw data from $\boldsymbol{D}$, the effect size and the $95 \% \mathrm{Cl}$.

production by inhibiting COX-1/COX-2 with intravenous indomethacin $(10 \mathrm{mg} / \mathrm{kg}) 30 \mathrm{~min}$ before LPS administration at ZT2 (Fig. $3 A$ ) prevented c-Fos expression in the VSAs [DH (Fig. 3D,E); NTS (Fig. 3F,G)], indicating that PGs are important mediators of the LPS-induced neuronal activation in the VSAs. Indomethacin alone did not affect the induction of c-Fos in the VSAs. PGE 2 is a type of PG that is highly inducible by LPS (Rotondo et al., 1988; Li et al., 2006). When we administered $P E_{2}(100 \mu \mathrm{g} / \mathrm{kg}$; iv) at $Z T 2$, we observed neuronal activation in the VSAs that resembled that of LPS [DH (Fig. 3D,E); NTS (Fig. 3F,G)], suggesting that $P G E_{2}$ could be a likely mediator of LPS. As previously reported (Sacco et al., 1998; Teeling et al., 2010), indomethacin treatment significantly increased LPS-induced TNF $\alpha$ plasma levels (Fig. 4A,B), suggesting that the sensing of PG by the VSAs contributes to the suppression of TNF $\alpha$. Furthermore, there was a significant increase in heart rate $30 \mathrm{~min}$ after the LPS administration (Fig. 4C), probably indicating a general sympathetic activation. The administration of indomethacin effectively prevented this increase in heart rate, suggesting that PGinduced neuronal activation in the VSAs in response to LPS, initiates an early sympathetic reflex that inhibits the secretion of TNF $\alpha$. To further investigate whether this reflex is sympathetic, we intravenously administered propranolol (Pro), a non-specific antagonist of $\beta$-adrenergic receptors, $30 \mathrm{~min}$ before the LPS administration. TNF $\alpha$ was increased after the adrenergic blockade (Fig. 4D,E), strongly suggesting that the efferent arm of the anti-inflammatory reflex comprises sympathetic nerves.

\section{Liver-spinal afferents start an anti-inflammatory reflex in response to LPS}

To investigate whether visceral sensory nerves transmit circulating immune information to the VSAs, we performed a peritoneal deafferentation with capsaicin. After deafferentation, LPS administration at ZT2 failed to induce c-Fos expression in the VSAs [DH (Fig. 5C,D); NTS (Fig. 5E,F)], indicating that the CNS receives circulating immune information via visceral sensory nerves. Compared with the sham animal, deafferented animals displayed increased TNF $\alpha$ levels after the LPS challenge (Fig. 5G,H), further suggesting that visceral sensory nerves initiate an inhibitory reflex. Basal TNF $\alpha$ levels were not increased in the deafferented animals, ruling out the possibility that capsaicin per se increases the inflammatory response.

Since c-Fos expression is upregulated in NTS and DH after LPS administration and the activation of both VSAs is prevented by peritoneal deafferentation, we needed to elucidate whether spinal and/or vagal sensory nerves 


\section{Sham+LPS}

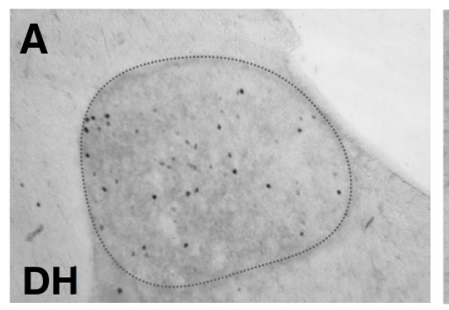

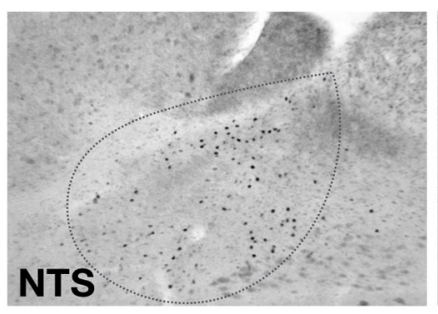

Caps+LPS

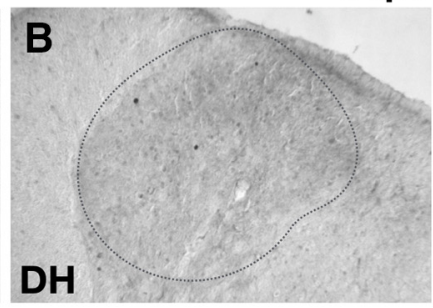

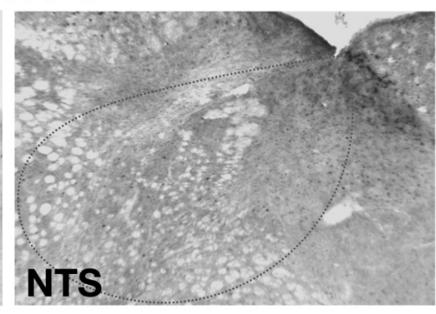

C

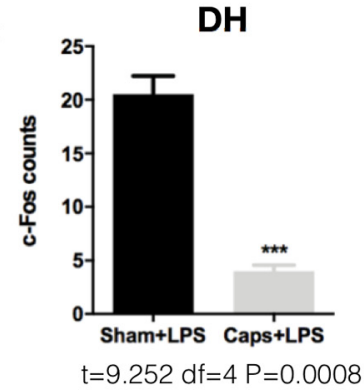

D

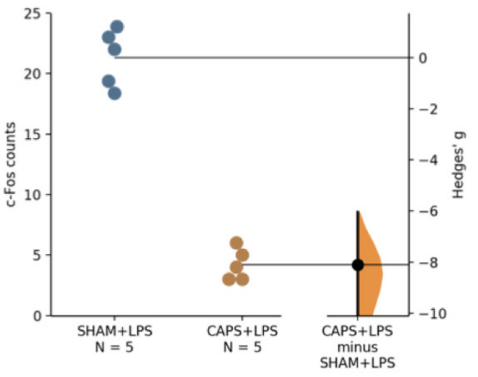

SHAM+LPS vs CAPS+LPS $=-8.11$ $[95.0 \% \mathrm{Cl}-10.2,-6.04] \mathrm{P}=0.0088$
E

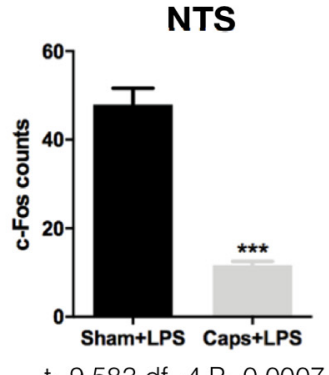

$\mathbf{F}$

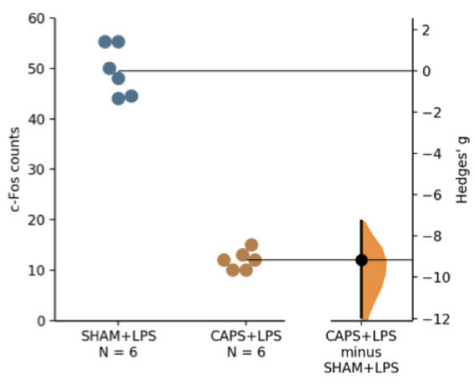

SHAM+LPS vs CAPS+LPS $=-9.28$

$[95.0 \% \mathrm{Cl}-14.4,-6.8] \mathrm{P}=0.0062$
G

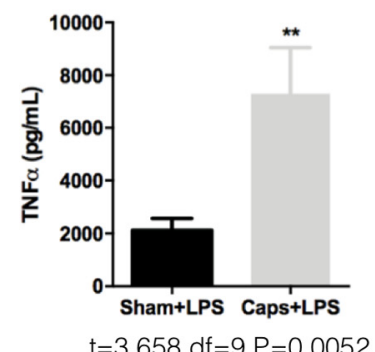

H

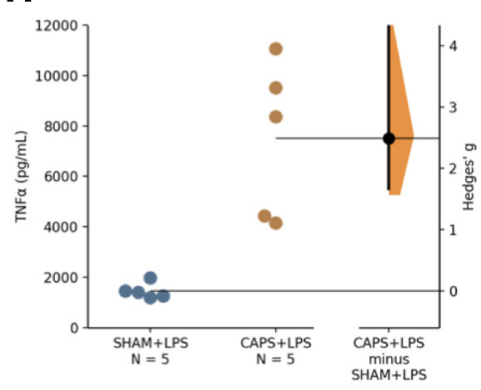

SHAM+LPS vs CAPS+LPS $=2.49$ $[95.0 \% \mathrm{Cl} 1.67,7.37] \mathrm{P}=0.003$.

Figure 5. Capsaicin-sensitive afferent nerves engage an anti-inflammatory reflex in response to LPS. Representative pictures of cFos expression in DH and NTS 50 min after LPS intravenous administration at ZT2 in $(\boldsymbol{A})$ sham animals (Sham + LPS) or (B) animals treated with intraperitoneal capsaicin (Caps+LPS). C, $\boldsymbol{E}$, Quantification of c-Fos immunoreactivity for each structure ( $n=5 /$ group). Data were analyzed with an unpaired two-tailed $t$ test; ${ }^{* \star *} p<0.001$ significance of difference against Sham + LPS. $\boldsymbol{D}, \boldsymbol{F}$, GardnerAltman estimation plot displaying the raw data from $\boldsymbol{C}, \boldsymbol{E}$, the effect size and the $95 \% \mathrm{Cl}$. $\mathbf{G}$, TNF $\alpha$ levels in plasma 50 min after LPS treatment. Data were analyzed with an unpaired two-tailed $t$ test; ${ }^{* *} p<0.01$ significance of difference against Sham + LPS. $\boldsymbol{H}$, Gardner-Altman estimation plot displaying the raw data from $\mathbf{G}$, the effect size and the $95 \% \mathrm{Cl}$.

were transmitting information necessary for the engagement of the anti-inflammatory reflex. Additionally, we needed to determine the visceral organ from which these afferent nerves originate. In contrast to the spleen, which lacks sensory innervation (Nance and Burns, 1989), the liver has both spinal and vagal sensory innervation (Yi et al., 2010) and plays an important role in the innate immune response (Jirillo et al., 2002). Therefore, we hypothesized that liver afferent nerves were responsible for transmitting peripheral immune information to the CNS.

To investigate this, we performed SLX or VLX and administered LPS at ZT2. Although c-Fos expression in the NTS was largely suppressed in the VLX animal (Fig. 6E,F), LPS-induced TNF $\alpha$ plasma concentration remained unchanged (Fig. 7A,B). These data show that vagal sensory input from the liver is dispensable for the reflex. In contrast, SLX effectively prevented LPS-induced neuronal activation in both VSAs [DH (Fig. 6C,D); NTS (Fig. 6E,F)] and significantly increased TNF $\alpha$ levels (Fig. 7A,B), similar to what happened after peritoneal deafferentation (Fig. $5 G, H)$. Taken together, liver-spinal afferents are responsible for activating the VSAs and are necessary components of the anti-inflammatory reflex.

Since the liver and spleen are the major sources of TNF $\alpha$ (Huston et al., 2006), the upregulated levels of plasma TNF $\alpha$ seen in the LPS-challenged SLX animals could be accompanied by TNF $\alpha$ content alterations in the liver and spleen. We expected an upregulation of TNF $\alpha$ in the liver because it was the denervated organ, yet to our surprise, we found that TNF $\alpha$ was significantly increased not only in the liver (Fig. 7C,D), but also in the spleen (Fig. 7E, $F$ ). We can conclude that the liver acts as an LPS sensor, but both liver and spleen serve as the effector organs for TNF $\alpha$ production/release. 


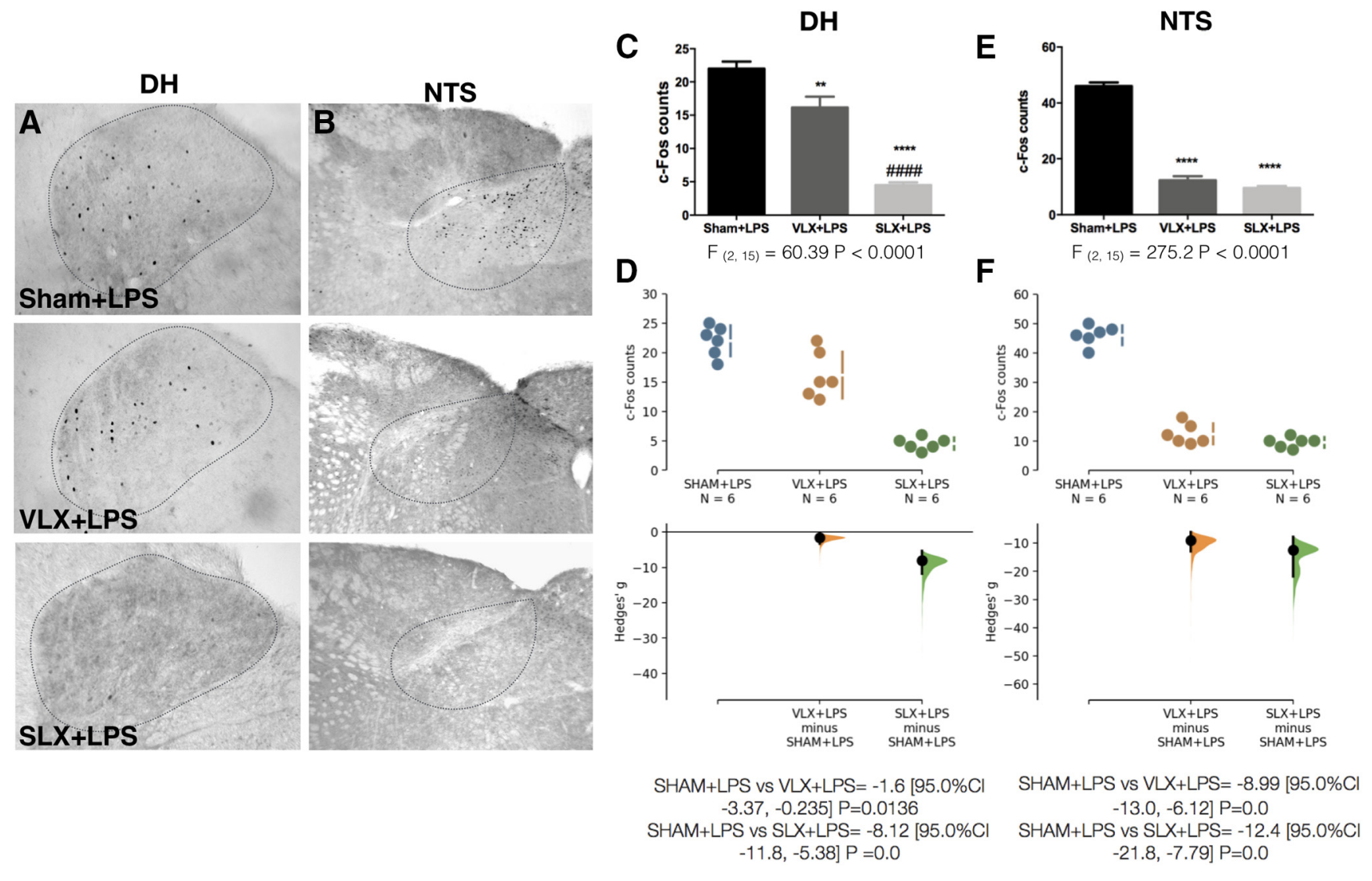

Figure 6. Liver-spinal afferents transmit the inflammatory signal to the VSAs. Representative pictures showing $\mathrm{C}-\mathrm{Fos}$ expression in (A) DH and (B) NTS 50 min after LPS intravenous administration at ZT2 in: sham (Sham +LPS), VLX (VLX+LPS), and SLX (SLX+LPS). C, E, Quantification of C-Fos immunoreactivity for each structure (n = 6/group). Data were analyzed with a one-way ANOVA followed by a Tukey's post hoc test; ${ }^{\star *} p<0.01,{ }^{* \star *} p<0.0001$, significance of difference against sham animal. $\boldsymbol{D}, \boldsymbol{F}$, Cumming estimation plot displaying the raw data from $\boldsymbol{C}$, $\boldsymbol{E}$, the effect size and the $95 \% \mathrm{Cl}$

\section{A time-of-day-dependent sensitivity to LPS in the VSAs shapes the TNF $\alpha$ release pattern from liver and spleen}

Another important aspect in the central control of inflammation is that the magnitude of the LPS-induced TNF $\alpha$ response depends on the time of the day, being significantly higher during the active period of the animal (Guerrero-Vargas et al., 2014; Fig. 8G,H). Considering our data, we hypothesized that a time-of-day-dependent sensing of LPS by the VSAs could account for the circadian variations in TNF $\alpha$ plasma concentration. To investigate this, we administered LPS at two different time points, $2 \mathrm{~h}$ after light onset (ZT2) and $2 \mathrm{~h}$ after light offset (ZT14). We found that when administered at ZT2, LPS induces a significantly higher c-Fos expression in both VSAs compared with ZT14 [DH (Fig. 8C,D); NTS (Fig. $8 E, F)]$. We hypothesized that $P G$ levels are upregulated at ZT2, contributing to the higher VSAs activation at this point. However, $\mathrm{PGE}_{2}$ plasma concentration at $\mathrm{ZT} 2$ and ZT14 did not differ (Fig. 8/), suggesting an increased sensitivity to LPS/PG in the VSAs at ZT2. Administering a higher dose of LPS $(100 \mu \mathrm{g} / \mathrm{kg})$ at ZT14 induced a neuronal activation like the one seen at ZT2 [DH (Fig. 9C,D); NTS (Fig. 9E,F)], demonstrating that a time-dependent sensitivity to LPS/PG promotes the different neuronal activation in the VSAs.

To further test our hypothesis that a time-of-daydependent sensing of LPS by the VSAs could account for the circadian variations in TNF $\alpha$ plasma concentration, we needed to evaluate other factors that could potentially contribute to the TNF $\alpha$ rhythm. One such factor is the diurnal variation in the expression of TLR4, therefore, we measured the expression of this receptor in the liver of intact animals at ZT2 and ZT14. We did not find a difference between TLR4 expression at these two time points (Fig. $9 G-I)$. Another possibility is that TNF $\alpha$ rhythm depends on light entrainment, hence the cytokine was measured under constant dark conditions. The TNF $\alpha$ rhythm persisted even in the absence of light (Fig. $9 \mathrm{~J}, K$ ), strengthening the view that the inflammatory response to LPS is indeed circadian regulated. Lastly, we reasoned that the circadian rhythm in plasma TNF $\alpha$ could be because of an oscillatory TNF $\alpha$ production by spleen and liver in response to LPS. We found that $50 \mathrm{~min}$ after LPS administration at ZT2, the TNF $\alpha$ content in the liver (Fig. 10A,B) and spleen (Fig. 10C,D) was elevated, but nearly undetectable when LPS was administered at ZT14. This pattern in TNF $\alpha$ levels is opposite to the one observed in 

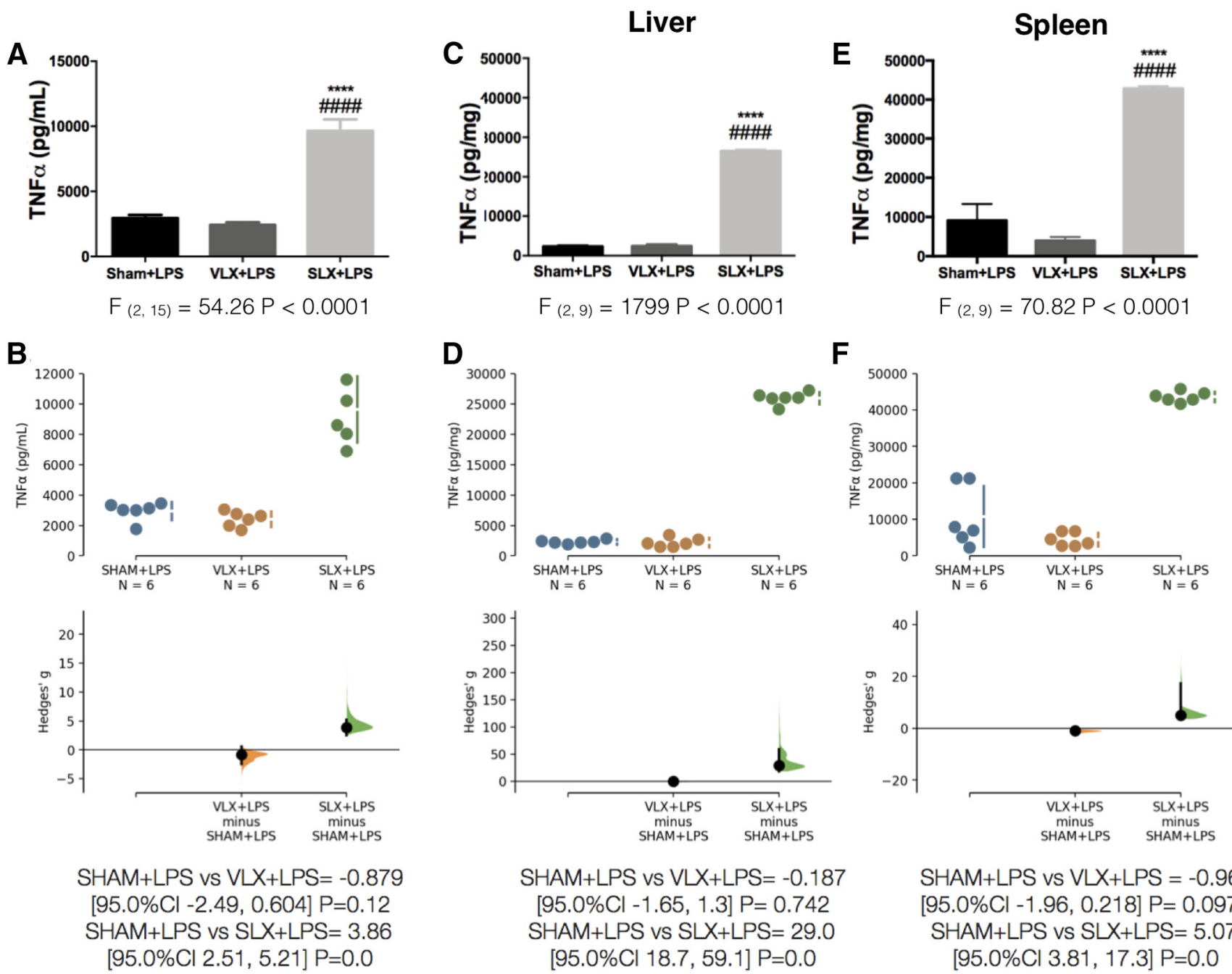

SHAM+LPS vs VLX+LPS $=-0.879$ $[95.0 \% \mathrm{Cl}-2.49,0.604] \mathrm{P}=0.12$ SHAM+LPS vs SLX+LPS $=3.86$ $[95.0 \% \mathrm{Cl} 2.51,5.21] \mathrm{P}=0.0$
SHAM+LPS vs VLX+LPS $=-0.962$ $[95.0 \% \mathrm{Cl}-1.96,0.218] \mathrm{P}=0.0972$ SHAM+LPS vs SLX+LPS $=5.07$ $[95.0 \% \mathrm{Cl} 3.81,17.3] \mathrm{P}=0.0$

Figure 7. Liver-spinal afferent nerves engage an anti-inflammatory reflex in response to LPS. TNF $\alpha$ quantification in: plasma $(\boldsymbol{A})$, liver $(\boldsymbol{C})$, and spleen $(\boldsymbol{E}) 50 \mathrm{~min}$ after LPS administration. Data from A, C, E were analyzed with a one-way ANOVA followed by a Tukey's post hoc test; ${ }^{* * *} p<0.0001$, significance of difference against sham animal; \#\#\#\#p $<0.0001$ significance of difference against VLX animal. $\boldsymbol{B}, \boldsymbol{D}, \boldsymbol{F}$, Cumming estimation plot displaying the raw data from $\boldsymbol{A}, \boldsymbol{C}, \boldsymbol{E}$, the effect size and the $95 \% \mathrm{Cl}$.

plasma (Fig. 8G,H), where TNF $\alpha$ is low at ZT2 and high at ZT14.

The administration of indomethacin has a time-of-daydependent ceiling-effect on LPS-induced TNF $\alpha$, given that this drug was able to further increase TNF $\alpha$ only during ZT2 (Figs. $4 A, B, 10 E, F$ ). This observation further suggests that the anti-inflammatory reflex is engaged only during the resting period and limits the maximal TNF $\alpha$ response to the active period.

We propose that the time-of-day-dependent gating of the incoming inflammatory signal in the VSAs engages an antiinflammatory reflex to promote the retention of $\mathrm{TNF} \alpha$ within the visceral organs at ZT2; explaining the low plasma $\mathrm{TNF} \alpha$ levels at this time point. In contrast, the low LPS/PG sensing in the VSAs during ZT14 does not activate the anti-inflammatory reflex, allowing the organs to freely release most of their TNF $\alpha$ content into circulation. This time-of-day-dependent pattern of TNF $\alpha$ release/retention may explain the circadian rhythm in plasma TNF $\alpha$ response to LPS.

\section{Discussion}

Earlier studies have proposed that CVOs play a role in the sensing of circulating LPS because of the expression of TLR4 in astrocytes (Laflamme and Rivest, 2001; Wuchert et al., 2008; Nakano et al., 2015). Indeed, the induction of cFos expression in the CVOs after LPS administration suggests their involvement (Elmquist et al., 1996; Buijs et al., 2008). However, the long survival times (2-6 h) used to evaluate neuronal activation do not exclude the possibility that LPS-induced cytokines, such as TNF $\alpha$ and IL-6, activate the CVOs. To address this issue, we used a shorter survival time $(50 \mathrm{~min})$ based on previous findings that indicate a cFos expression peak 40-60 min after a stimulus (Sharp et al., 1991; Hoffman et al., 1993; Lin et al., 2011; Zhong et al., 2014). Therefore, we can conclude that the CVOs do not contribute to the early-phase sensing of LPS.

In agreement with our observations, CVOs show a very low basal expression of the mRNA of p55 and p75 TNF $\alpha$ receptors and this expression is upregulated 3-6 $\mathrm{h}$ after 
LPS ZT2

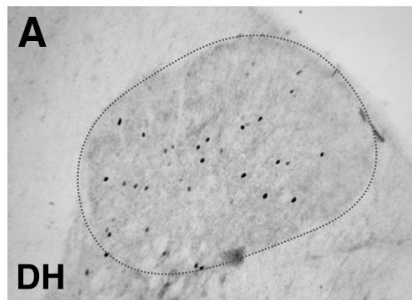

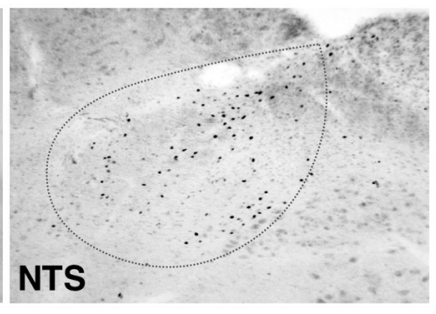

LPS ZT14

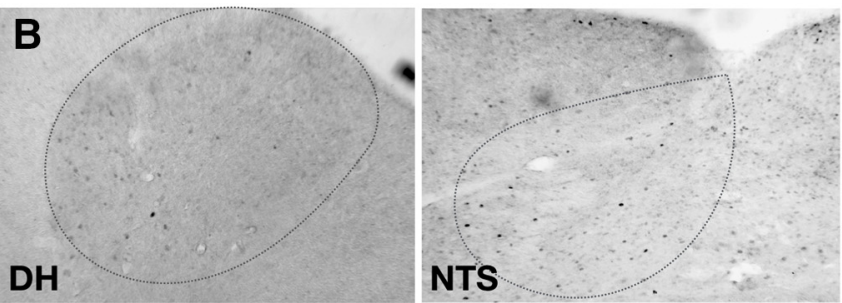

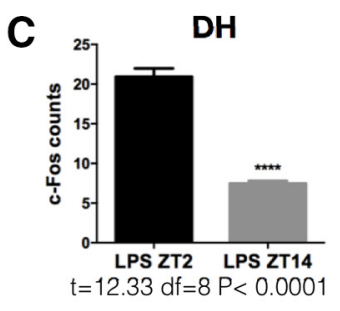

D

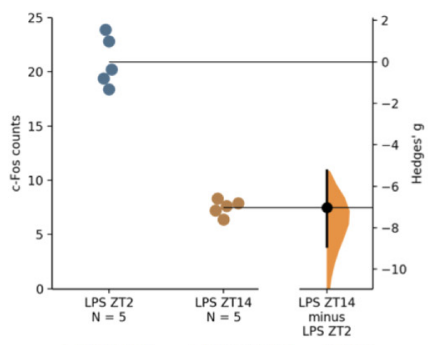

LPS ZT2 vs LPS ZT14 $=-7.04$ $[95.0 \% \mathrm{Cl}-8.91,-5.26] \mathrm{P}=0.0066$
E

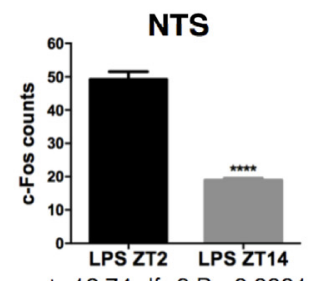

$t=12.74 d f=8 \quad P<0.0001$

$\mathbf{F}$

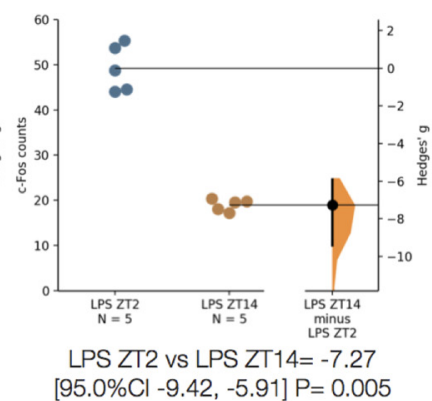

G

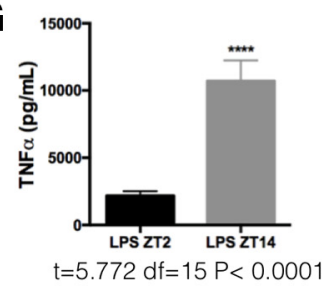

H

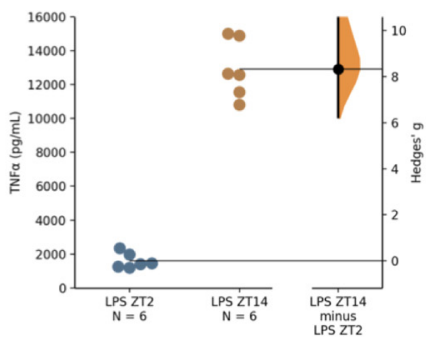

LPS ZT 2 vs LPS ZT14 $=8.32$ $[95.0 \% \mathrm{Cl} 6.24,10.8] \mathrm{P}=0.0008$

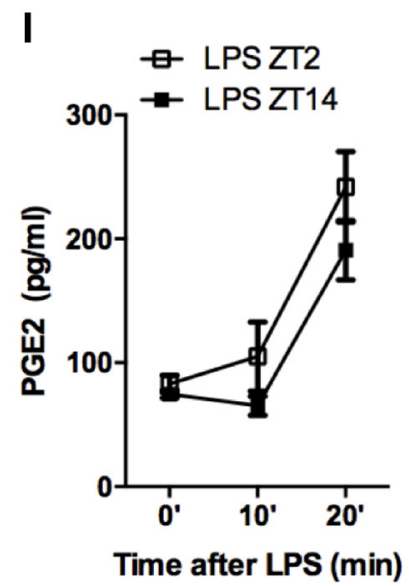

Interaction

$F_{(2,8)}=1.070 P=0.3873$

Figure 8. VSAs display a time-of-day-dependent activation in response to LPS. Representative pictures of c-Fos expression in DH and NTS $50 \mathrm{~min}$ after LPS intravenous administration at two time points: $(\boldsymbol{A})$ ZT2 (2 h after light onset) or (B) ZT14 (2 h after lights off). $\boldsymbol{C}, \boldsymbol{E}$, Quantification of c-Fos immunoreactivity for each structure ( $n=5 /$ group). Data were analyzed with an unpaired two-tailed $t$ test; ${ }^{\star \star \star \star} p<0.0001$, significance of difference between LPS ZT2 and LPS ZT14. D, F, Gardner-Altman estimation plot displaying the raw data from $\boldsymbol{C}, \boldsymbol{E}$, the effect size and the $95 \% \mathrm{Cl}$. G, TNF $\alpha$ levels in plasma 50 min after LPS intravenous administration at ZT2 or ZT14 ( $n=5$ /group). Data were analyzed with an unpaired two-tailed $t$ test; ${ }^{\star * \star *} p<0.0001$, significance of difference between LPS ZT2 and LPS ZT14. $\boldsymbol{H}$, Gardner-Altman estimation plot displaying the raw data from $\mathbf{G}$, the effect size and the $95 \% \mathrm{Cl}$. $\boldsymbol{I}$, PGE 2 levels in plasma after LPS intravenous administration at ZT2 or ZT4 ( $n=5 /$ group). Data were analyzed with a two-way ANOVA for repeated measures followed by a Sidak's post hoc test.

intraperitoneal administration of LPS or after intravenous administration of TNF $\alpha$ (Nadeau and Rivest, 1999). Consequently, any cytokine signaling to the CVOs most likely occurs in a late-phase of the LPS challenge. This agrees with the proposal that the signaling to the CVOs could account for delayed responses to LPS, such as fever (Blatteis, 1992; Takahashi et al., 1997).

We also tested the possibility that LPS is sensed by the vagus nerve. After cutting the hepatic branch of the vagus, TNF $\alpha$ levels in plasma remained largely unchanged, which indicates that the vagus does not start a reflex to inhibit inflammation. On the other hand, existing literature proposes that proinflammatory cytokines such as IL1 $\beta$ can signal the inflammatory status of the body through the vagus nerve (Ek et al., 1998; Hansen et al., 2001; Zanos et al., 2018). However, as aforementioned, any vagal cytokine sensing may only occur $\sim 30 \mathrm{~min}$ after the LPS challenge, when cytokines appear in circulation, and it is therefore not detected in the present study.
An important factor to consider for the interpretation of our data are the dose of LPS. Other studies have used doses of LPS 50-500 times higher than the dose used here. We are aware that according to the dose and the administration route, other effects can be and have been observed. A previous study by our group (Guerrero-Vargas et al., 2014) evaluated two doses of LPS, 100 and $2 \mu \mathrm{g} / \mathrm{kg}$. The higher dose proved to be lethal under certain experimental conditions and therefore, a dose of $2 \mu \mathrm{g} / \mathrm{kg}$ was chosen for the present study. This dose effectively activates the immune response and allows a careful dissection of the LPS transmission pathway without overwhelming the system.

The data obtained from the experiments where indomethacin was used indicate that LPS-induced PG are largely responsible for the signaling of inflammatory information to the CNS. One important consideration is that the action of indomethacin is not tissue or cell specific. It has been shown that $\sim 80 \%$ of intravenous injected LPS 
Low LPS ZT14

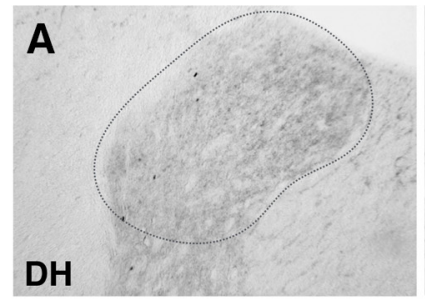

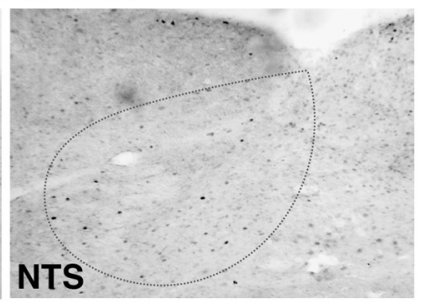

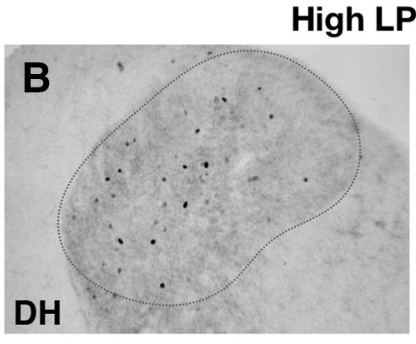

NTS
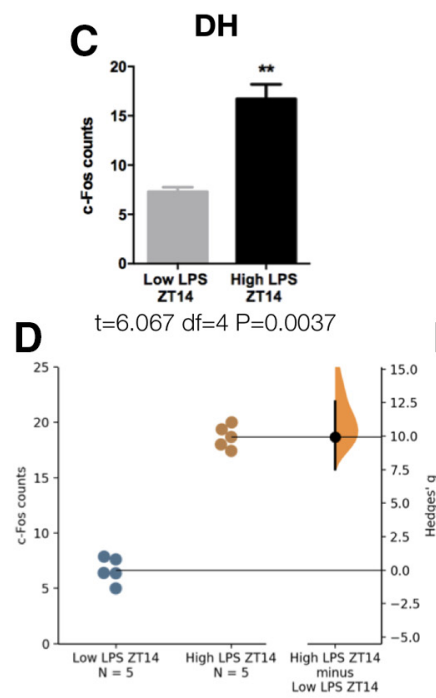

Low LPS ZT14 vs High LPS ZT14=9.94 [95.0\%Cl 7.51, 12.6] $\mathrm{P}=0.0042$
E

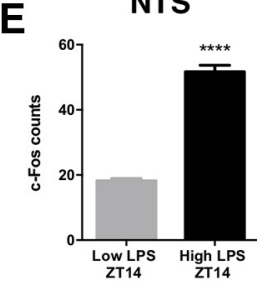

$\mathbf{F}$

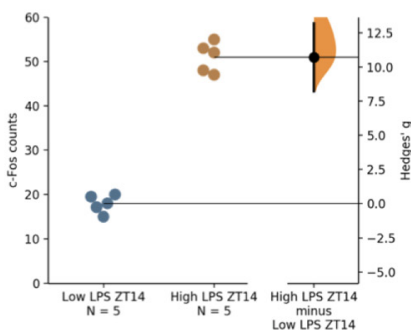

Low LPS ZT14 vs High LPS ZT14=10.7 [95.0\%Cl 8.23, 13.2] $\mathrm{P}=0.0$

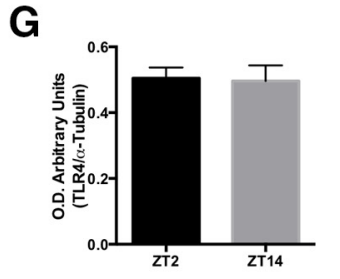

H

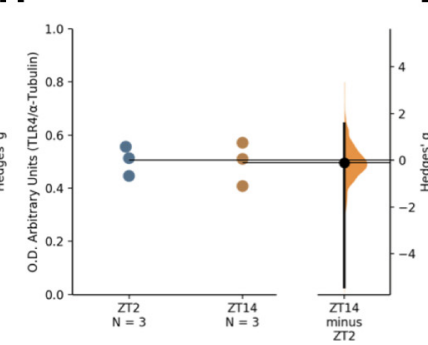

ZT2 vs $Z T 14=-0.0985[95.0 \% \mathrm{Cl}$ $-5.42,1.57] \mathrm{P}=0.809$

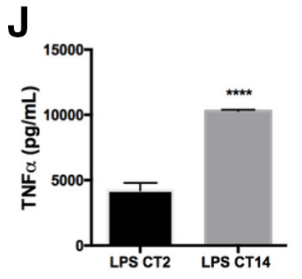

K

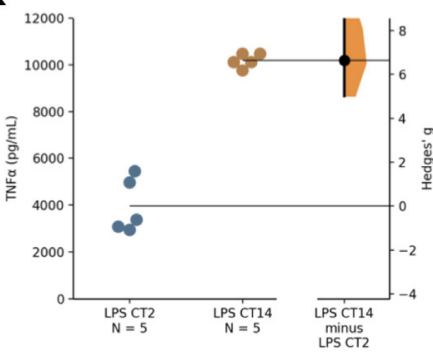

LPS CT2 vs LPS CT14 $=6.64$ $[95.0 \% \mathrm{Cl} 5.01,9.7] \mathrm{P}=0.0016$

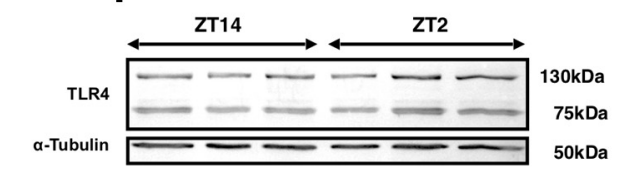

Figure 9. VSAs display a time-of-day-dependent sensitivity to circulating LPS. Representative pictures of c-Fos expression in DH and NTS $50 \mathrm{~min}$ after $(\boldsymbol{A})$ low LPS $(2 \mu \mathrm{g} / \mathrm{kg})$ or $(\boldsymbol{B})$ high LPS $(100 \mu \mathrm{g} / \mathrm{kg})$ at ZT14. $\boldsymbol{C}$, $\boldsymbol{E}$, Quantification of c-Fos immunoreactivity for each structure ( $n=5 /$ group). Data were analyzed with an unpaired two-tailed $t$ test; ${ }^{* *} p<0.01$, ${ }^{* * *} p<0.0001$ significance of difference between low LPS and high LPS. $\boldsymbol{D}, \boldsymbol{F}$, Gardner-Altman estimation plot displaying the raw data from $\boldsymbol{C}, \boldsymbol{E}$, the effect size and the $95 \% \mathrm{Cl}$. G, Quantification of Western blotting for TLR4 relative expression at ZT2 and ZT14 in intact animals ( $n=3 /$ group). Data were analyzed with an unpaired two-tailed $t$ test. $\boldsymbol{H}$, Gardner-Altman estimation plot displaying the raw data from $\boldsymbol{G}$, the effect size and the $95 \% \mathrm{Cl}$. I, Representative image of the TLR4 Western blotting. $\boldsymbol{J}$, TNF $\alpha$ levels in plasma 50 min after LPS intravenous administration at CT2 or CT14 ( $n=5 /$ group). Data were analyzed with an unpaired two-tailed $t$ test; ${ }^{\star \star \star *} p<0.0001$, significance of difference between LPS ZT2 and LPS ZT14. K, Gardner-Altman estimation plot displaying the raw data from $\boldsymbol{J}$, the effect size and the $95 \% \mathrm{Cl}$.

accumulates in the liver within the first hours after its administration (Jirillo et al., 2002). This visceral organ has an important population of resident macrophages, the Kupffer cells, which could immediately detect the LPS and start producing early proinflammatory molecules such as PG and later cytokines (Li et al., 2006). We hypothesize that indomethacin mainly prevents the production of PG by the liver, however, we have not proven it. PG can also be produced within the CNS (Saper and Breder, 1992), but they need a signal to be synthesized. As previously mentioned, LPS minimally crosses the BBB (Banks and Robinson, 2010), and we have shown that LPS does not activate the CVOs, the areas where PG most likely are synthesized and exert major effects (Takahashi et al.,
1997). Therefore, we believe that the production of $P G$ by the liver is the most relevant for the initiation of the anti-inflammatory reflex.

\section{The liver-spinal axis}

We demonstrate the existence of a circuit comprising two crucial aspects. The first element involves the liverspinal visceral afferents, herein termed as the liver-spinal axis, which is responsible for alerting the CNS about circulating LPS by sensing PG. The fact that the liver is involved in the early transmission of circulating immune information can be expected, since via its portal system, where blood from the gut is received, the liver is the first 
A

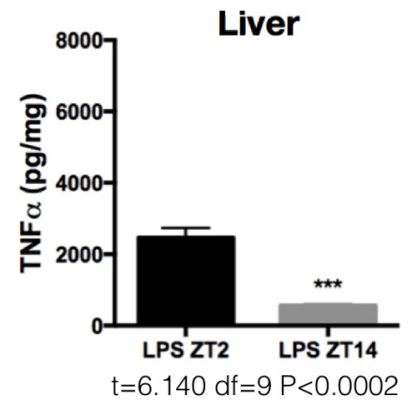

B

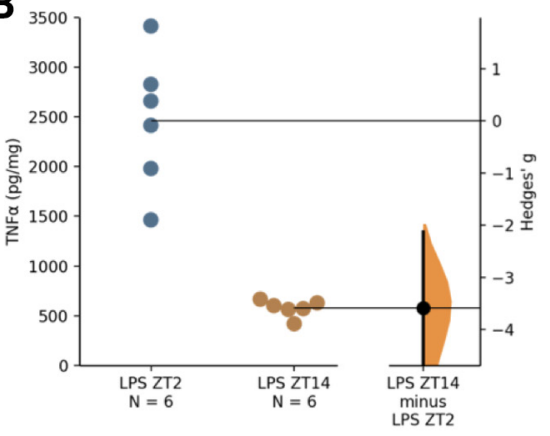

LPS ZT2 vS LPS ZT14= -3.59

$[95.0 \% \mathrm{Cl}-6.24,-2.13] \mathrm{P}=0.0008$
C

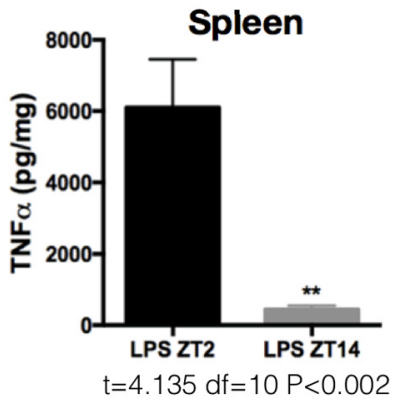

D

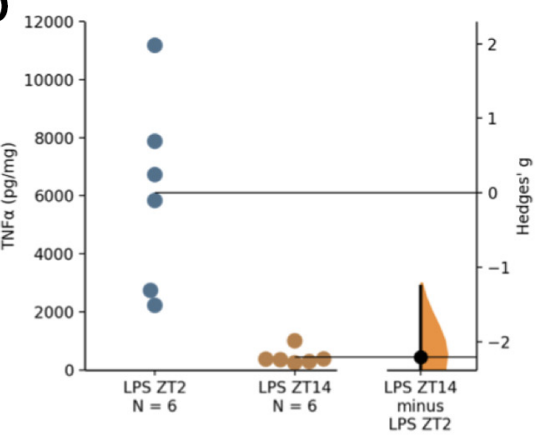

LPS ZT2 vs LPS ZT14= -2.2 $[95.0 \% \mathrm{Cl}-3.76,-1.26] \mathrm{P}=0.0$
E

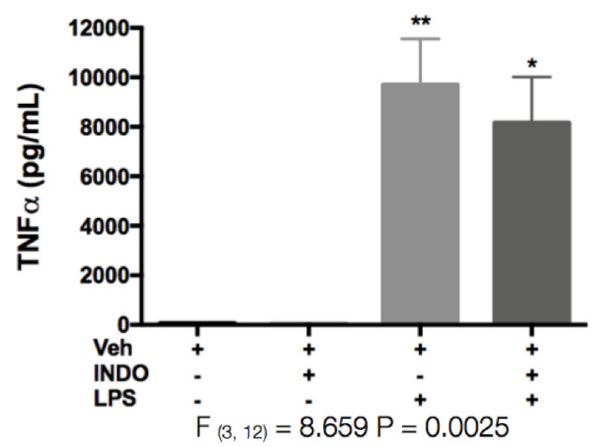

F

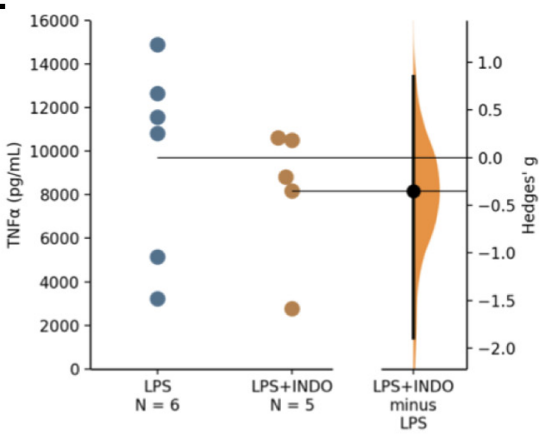

LPS vs LPS $+\mid N D O=-0.352$

$[95.0 \% \mathrm{Cl}-1.89,0.851] \mathrm{P}=0.529$

Figure 10. A time-of-day-dependent TNF $\alpha$ release pattern from liver and spleen shapes the rhythm of plasma TNF $\alpha$. TNF $\alpha$ levels in liver (A) and spleen (C) 50 min after LPS intravenous administration at ZT2 or ZT14. Data were analyzed with an unpaired two-tailed $t$ test $\left(n=5 /\right.$ group); ${ }^{* \star} p<0.01$, ${ }^{\star \star \star} p<0.001$, significance of difference between LPS ZT2 and LPS ZT14. B, $\boldsymbol{D}$, Gardner-Altman estimation plot displaying the raw data from $\boldsymbol{A}, \boldsymbol{C}$, the effect size and the $95 \% \mathrm{Cl}$. $\boldsymbol{E}$, TNF $\alpha$ levels in plasma 50 min after LPS or LPS+Indo administration at ZT14. Data were analyzed with a one-way ANOVA followed by a Tukey's post hoc test; ${ }^{*} p<0.05$, ${ }^{* *} p$ $<0.01$ significance of difference against Veh. $\boldsymbol{F}$, Gardner-Altman estimation plot displaying the raw data from $\boldsymbol{E}$, the effect size and the $95 \% \mathrm{Cl}$.

organ to get exposed to pathogens or toxins ingested with food intake (Jirillo et al., 2002).

We propose this is a spinal reflex since consistently throughout our different experimental conditions, the decrease in c-Fos expression in the $\mathrm{DH}$ of the spinal cord was always accompanied by higher circulating TNF $\alpha$ levels. These data strongly suggest that the higher neuronal activation in the DH seen at ZT2 serves to prevent a high inflammatory process. The same did not happen in the NTS, since the loss of its activation in the VLX condition was not accompanied by an increase in TNF $\alpha$ levels. Spinal afferent nerves are normally associated with sensorimotor reflexes and nociceptive pathways. Our study shows that the spinal cord can use similar pathways to act on the inflammatory process, which is consistent with other studies that have reported the expression of various TLRs, including 4, 3, and 7, in visceral sensory neurons of the DRG (Barajon et al., 2009; Ochoa-Cortes et al., 2010). The circuit we describe may not completely fulfill the definition of a classical spinal reflex, which implies that the circuit should be complete only within the spinal cord. In the present study we show that this reflex also involves areas outside of the spinal cord, particularly the NTS, which is located in the brainstem. As for the circuits involved within the spinal cord, previous retrograde viral tracing studies following the sympathetic branch of the liver, showed tracing of the sympathetic motor neurons in the intermediolateral column of the spinal cord (IML), followed by tracing of the neurons in the $\mathrm{DH}$; indicating that such direct spinal-sympathetic reflex is possible (Kalsbeek et al., 2004).

The use of capsaicin to ablate afferent spinal nerves close to the portal system could also compromise vagal afferents, which opens the question of whether vagal afferents also play a role in the early signaling of LPS. This possibility could also potentially explain the loss of NTS activation after the SLX denervation. Nonetheless, removing the hepatic branch of the vagus nerve did not prevent c-Fos activation in both VSAs as observed after the SLX denervation. Moreover, vagal denervation had no effect on TNF $\alpha$ levels, which is strong evidence to conclude that spinal and not vagal visceral afferents are responsible for the anti-inflammatory reflex observed in the present study.

\section{Time-of-day-dependent anti-inflammatory reflex}

The second crucial aspect of the described pathway is its rhythmicity. We show that the sensitivity of the VSAs to incoming inflammatory input is time-of-day-dependent, whereby the VSAs receive this information only during the 
resting period; preventing a strong inflammatory process at this point. In contrast, when the VSAs do not receive this information during the active period, the anti-inflammatory process is not initiated; resulting in higher circulating TNF $\alpha$ levels. The fact that pain also shows a circadian rhythm (Pickard, 1987; Konecka and Sroczynska, 1998; Zhang et al., 2012) is in agreement with our data, indicating that the circadian system may render visceral sensory neurons more or less sensitive to stimuli, including inflammatory signals.

The rhythm in the TNF $\alpha$ response to LPS has been previously observed and its circadian regulation is evidenced by the fact that lesioning the central clock, the suprachiasmatic nucleus, results in an exacerbated inflammatory response to LPS (Guerrero-Vargas et al., 2014). In addition, desynchronization also promotes an exacerbated inflammatory response to LPS (Guerrero-Vargas et al., 2015). Our data propose a mechanism for the circadian control of the TNF $\alpha$ response. A time-of-day-dependent activity of the liver-spinal axis promotes the retention of TNF $\alpha$ in the liver and spleen during the resting period, promoting a lower plasma TNF $\alpha$ level at this point.

One factor that could also influence the rhythmicity of $\mathrm{TNF} \alpha$ is the endogenous rhythm of corticosterone. We have previously shown that the corticosterone levels at ZT14 are considerably higher than those observed at ZT2 (Kalsbeek et al., 1996). However, corticosterone is known to have anti-inflammatory effects (Smoak and Cidlowski, 2004; Baschant and Tuckermann, 2010). Therefore, the high corticosterone levels observed at ZT14 do not agree with the high levels of TNF $\alpha$ observed at this time point. Similarly, the low levels of corticosterone observed at ZT2 should promote higher TNF $\alpha$ levels, but that is not the case. For this reason, it is unlikely that the circadian rhythm in corticosterone contributes to the TNF $\alpha$ rhythm.

$\mathrm{TNF} \alpha$ is synthesized as transmembrane pro-TNF $\alpha$ and is cleaved from the cell membrane by the metalloprotease ADAM17 (also known as TNF $\alpha$ converting enzyme; Bell et al., 2007). Observations in primary cultures of cortical neurons showed that noradrenaline can decrease the enzymatic activity of ADAM17 (Madrigal et al., 2017). Our data suggest that the anti-inflammatory reflex is sympathetic because of its impairment by propranolol. Therefore, we hypothesize that the function of ADAM17 is hampered by noradrenaline released by the anti-inflammatory reflex during the resting period, thus accounting for the accumulation of TNF $\alpha$ in liver and spleen.

Since the probability of encountering a pathogen is higher during the active period than during the resting period, the time-of-day-dependent activation of the liverspinal axis could be an adaptive mechanism to narrow the time-window of the inflammatory response to when it is most likely needed.

In conclusion, the present study describes a novel pathway by which the anti-inflammatory reflex is engaged after an LPS challenge: the liver-spinal axis. The early detection of circulating LPS is conducted by spinal visceral afferents and not by the CVOs nor vagal visceral afferents. In addition, this sensing is largely dependent on the presence of PGs. Finally, we prove that the sensing of LPS by the liver-spinal axis is time-of-day dependent and shapes the circadian rhythm of TNF $\alpha$.

\section{References}

Banks WA, Robinson SM (2010) Minimal penetration of lipopolysaccharide across the murine blood-brain barrier. Brain Behav Immun 24:102-109.

Barajon I, Serrao G, Arnaboldi F, Opizzi E, Ripamonti G, Balsari A, Rumio C (2009) Toll-like receptors 3, 4, and 7 are expressed in the enteric nervous system and dorsal root ganglia. J Histochem Cytochem 57:1013-1023.

Baschant U, Tuckermann J (2010) The role of the glucocorticoid receptor in inflammation and immunity. J Steroid Biochem Mol Biol 120:69-75.

Baud V, Karin M (2001) Signal transduction by tumor necrosis factor and its relatives. Trends Cell Biol 11:372-377.

Bell JH, Herrera AH, Li Y, Walcheck B (2007) Role of ADAM17 in the ectodomain shedding of TNF- $\alpha$ and its receptors by neutrophils and macrophages. J Leukoc Biol 82:173-176.

Blatteis CM (1992) Role of the OVLT in the febrile response to circulating pyrogens. Prog Brain Res 91:409-412.

Borovikova LV, Ivanova S, Zhang M, Yang H, Botchkina GI, Watkins LR, Wang H, Abumrad N, Eaton JW, Tracey KJ (2000) Vagus nerve stimulation attenuates the systemic inflammatory response to endotoxin. Nature 405:458-462.

Bret-Dibat JL, Creminon C, Couraud JY, Kelley KW, Dantzer R, Kent S (1997) Systemic capsaicin pretreatment fails to block the decrease in food-motivated behavior induced by lipopolysaccharide and interleukin-1 $\beta$. Brain Res Bull 42:443-449.

Browning KN, Verheijden S, Boeckxstaens GE (2017) The vagus nerve in appetite regulation, mood, and intestinal inflammation. Gastroenterology 152:730-744.

Buijs RM, Van der Vliet J, Garidou ML, Huitinga I, Escobar C (2008) Spleen vagal denervation inhibits the production of antibodies to circulating antigens. PLoS One 3:e3152.

Dantzer R (2001) Cytokine-induced sickness behavior: mechanisms and implications. Ann NY Acad Sci 933:222-234.

Dantzer R, O'Connor JC, Freund GG, Johnson RW, Kelley KW (2008) From inflammation to sickness and depression: when the immune system subjugates the brain. Nat Rev Neurosci 9:46-56.

Druzd D, Matveeva O, Ince L, Harrison U, He W, Schmal C, Herzel H, Tsang AH, Kawakami N, Leliavski A, Uhl O, Yao L, Sander LE, Chen CS, Kraus K, de Juan A, Hergenhan SM, Ehlers M, Koletzko B, Haas R, et al. (2017) Lymphocyte circadian clocks control lymph node trafficking and adaptive immune responses. Immunity 46:120-132.

Ek M, Kurosawa M, Lundeberg T, Ericsson A (1998) Activation of vagal afferents after intravenous injection of interleukin- $1 \beta$ : role of endogenous prostaglandins. J Neurosci 18:9471-9479.

Elmquist JK, Scammell TE, Jacobson CD, Saper CB (1996) Distribution of Fos-like immunoreactivity in the rat brain following intravenous lipopolysaccharide administration. J Comp Neurol 371:85-103.

Fujita S, Bohland M, Sanchez-Watts G, Watts AG, Donovan CM (2007) Hypoglycemic detection at the portal vein is mediated by capsaicin-sensitive primary sensory neurons. Am J Physiol Endocrinol Metab 293:96-101.

Guerrero-Vargas NN, Salgado-Delgado R, Basualdo MDC, García J, Guzmán-Ruiz M, Carrero JC, Escobar C, Buijs RM (2014) Reciprocal interaction between the suprachiasmatic nucleus and the immune system tunes down the inflammatory response to lipopolysaccharide. J Neuroimmunol 273:22-30.

Guerrero-Vargas NN, Guzmán-Ruiz M, Fuentes R, García J, Salgado-Delgado R, Basualdo M del C, Escobar C, Markus RP, Buijs RM (2015) Shift work in rats results in increased inflammatory response after lipopolysaccharide administration: a role for food consumption. J Biol Rhythms 30:318-330. 
Hansen MK, O'Connor KA, Goehler LE, Watkins LR, Maier SF (2001) The contribution of the vagus nerve in interleukin- $1 \beta$-induced fever is dependent on dose. Am J Physiol Regul Integr Comp Physiol 280:R929-R934.

Ho J, Tumkaya T, Aryal S, Choi H, Claridge-Chang A (2019) Moving beyond $P$ values: data analysis with estimation graphics. Nat Methods 16:565-566.

Hoffman GE, Smith MS, Verbalis JG (1993) c-Fos and related immediate early gene products as markers of activity in neuroendocrine systems. Front Neuroendocrinol 14:173-213.

Hosoi T, Okuma Y, Matsuda T, Nomura Y (2005) Novel pathway for LPS-induced afferent vagus nerve activation: possible role of nodose ganglion. Auton Neurosci 120:104-107.

Huston JM, Ochani M, Rosas-Ballina M, Liao H, Ochani K, Pavlov VA, Gallowitsch-Puerta M, Ashok M, Czura CJ, Foxwell B, Tracey KJ, Ulloa L (2006) Splenectomy inactivates the cholinergic antiinflammatory pathway during lethal endotoxemia and polymicrobial sepsis. J Exp Med 203:1623-1628.

limuro Y, Nishiura T, Hellerbrand C, Behrns KE, Schoonhoven R, Grisham JW, Brenner DA (1998) NF $\kappa$ appaB prevents apoptosis and liver dysfunction during liver regeneration. J Clin Invest 101:802-811.

Jirillo E, Caccavo D, Magrone T, Piccigallo E, Amati L, Lembo A, Kalis C, Gumenscheimer M (2002) The role of the liver in the response to LPS: experimental and clinical findings. J Endotoxin Res 8:319-327.

Kalsbeek A, van Heerikhuize JJ, Wortel J, Buijs RM (1996) A diurnal rhythm of stimulatory input to the hypothalamo-pituitary-adrenal system as revealed by timed intrahypothalamic administration of the vasopressin V1 antagonist. J Neurosci 16:5555-5565.

Kalsbeek A, La Fleur S, Van Heijningen C, Buijs RM (2004) Suprachiasmatic GABAergic Inputs to the Paraventricular Nucleus Control Plasma Glucose Concentrations in the Rat via Sympathetic Innervation of the Liver. J Neurosci 24:7604-7613.

Kalsbeek A, Liu J, Lei J, Timmermans L, Foppen E, Cailotto C, Fliers $E$ (2012) Differential involvement of the suprachiasmatic nucleus in lipopolysaccharide-induced plasma glucose and corticosterone responses. Chronobiol Int 29:835-849.

Konecka AM, Sroczynska I (1998) Circadian rhythm of pain in male mice. Gen Pharmacol 31:809-810.

Laflamme N, Rivest S (2001) Toll-like receptor 4: the missing link of the cerebral innate immune response triggered by circulating gram-negative bacterial cell wall components. FASEB J 15:155163.

Li Z, Perlik V, Feleder C, Tang Y, Blatteis CM (2006) Kupffer cell-generated PGE2 triggers the febrile response of guinea pigs to intravenously injected LPS. Am J Physiol Regul Integr Comp Physiol 290:1262-1270.

Lin D, Boyle MP, Dollar P, Lee H, Lein ES, Perona P, Anderson DJ (2011) Functional identification of an aggression locus in the mouse hypothalamus. Nature 470:221-227.

Lukacs NW, Strieter RM, Chensue SW, Widmer M, Kunkel SL (1995) TNF-alpha mediates recruitment of neutrophils and eosinophils during airway inflammation. J Immunol 154:5411-5417.

Madrigal JLM, Caso JR, García-Bueno B, Gutiérrez IL, Leza JC (2017) Noradrenaline induces CX3CL1 production and release by neurons. Neuropharmacology 114:146-155.

Martelli D, Yao ST, McKinley MJ, McAllen RM (2014) Reflex control of inflammation by sympathetic nerves, not the vagus. J Physiol 592:1677-1686.

Méndez-Ferrer S, Lucas D, Battista M, Frenette PS (2008) Haematopoietic stem cell release is regulated by circadian oscillations. Nature 452:442-447.

Nadeau S, Rivest S (1999) Effects of circulating tumor necrosis factor on the neuronal activity and expression of the genes encoding the tumor necrosis factor receptors (p55 and p75) in the rat brain: a view from the blood-brain barrier. Neuroscience 93:1449-1464.
Nakano Y, Furube E, Morita S, Wanaka A, Nakashima T, Miyata S (2015) Astrocytic TLR4 expression and LPS-induced nuclear translocation of STAT3 in the sensory circumventricular organs of adult mouse brain. J Neuroimmunol 278:144-158.

Nance M, Burns J (1989) Innervation of the spleen in the rat: evidence for absence of afferent innervation. Brain Behav Immun 3:281-290.

Ochoa-Cortes F, Ramos-Lomas T, Miranda-Morales M, Spreadbury I, Ibeakanma C, Barajas-Lopez C, Vanner S (2010) Bacterial cell products signal to mouse colonic nociceptive dorsal root ganglia neurons. Am J Physiol Gastrointest Liver Physiol 299:G723-G732.

Ott D, Murgott J, Rafalzik S, Wuchert F, Schmalenbeck B, Roth J, Gerstberger R (2010) Neurons and glial cells of the rat organum vasculosum laminae terminalis directly respond to lipopolysaccharide and pyrogenic cytokines. Brain Res 1363:93-106.

Paxinos G, Watson C (2005) The Rat Brain in Stereotaxic Coordinates, Elsevier Academic Press.

Pickard GE (1987) Circadian rhythm of nociception in the golden hamster. Brain Res 425:395-400.

Rotondo D, Abul HT, Milton AS, Davidson J (1988) Pyrogenic immunomodulators increase the level of prostaglandin E2 in the blood simultaneously with the onset of fever. Eur J Pharmacol 154:145-152.

Sacco S, Agnello D, Sottocorno M, Lozza G, Monopoli A, Villa P, Ghezzi P (1998) Nonsteroidal anti-inflammatory drugs increase tumor necrosis factor production in the periphery but not in the central nervous system in mice and rats. J Neurochem 71:2063-2070.

Saper CB, Breder CD (1992) Endogenous pyrogens in the CNS: role in the febrile response. Prog Brain Res 93:419-429.

Scheiermann C, Kunisaki Y, Lucas D, Chow A, Jang JE, Zhang D, Hashimoto D, Merad M, Frenette PS (2012) Adrenergic nerves govern circadian leukocyte recruitment to tissues. Immunity 37:290-301.

Sharp FF, Hisanaga K, Sagar M, Hicks K, Lowenstein D (1991) c-fos mRNA, Fos, and Fos-related antigen induction by hypertonic saline and stress. J Neurosci 11:2321-2331.

Smoak KA, Cidlowski JA (2004) Mechanisms of glucocorticoid receptor signaling during inflammation. Mech Ageing Dev 125:697706.

Takahashi Y, Smith P, Ferguson A, Pittman QJ (1997) Circumventricular organs and fever. Am J Physiol Regul Integr Comp Physiol 273:1690-1695.

Teeling JL, Cunningham C, Newman TA, Perry VH (2010) The effect of non-steroidal anti-inflammatory agents on behavioural changes and cytokine production following systemic inflammation: implications for a role of COX-1. Brain Behav Immun 24:409-419.

Tognini P, Thaiss CA, Elinav E, Sassone-Corsi P (2017) Circadian coordination of antimicrobial responses. Cell Host Microbe 22:185192.

Watson C, Paxinos G, Kayalioglu G, Heise C (2009) Atlas of the rat spinal cord. San Diego: Elsevier Ltd.

Wuchert F, Ott D, Murgott J, Rafalzik S, Hitzel N, Roth J, Gerstberger R (2008) Rat area postrema microglial cells act as sensors for the toll-like receptor-4 agonist lipopolysaccharide. J Neuroimmunol 204:66-74.

Yi CX, la Fleur SE, Fliers E, Kalsbeek A (2010) The role of the autonomic nervous liver innervation in the control of energy metabolism. Biochim Biophys Acta 1802:416-431.

Zanos TP, Silverman HA, Levy T, Tsaava T, Battinelli E, Lorraine PW, Ashe JM, Chavan SS, Tracey KJ, Bouton CE (2018) Identification of cytokine-specific sensory neural signals by decoding murine vagus nerve activity. Proc Natl Acad Sci USA 115:E4843-E4852.

Zhang J, Li H, Teng H, Zhang T, Luo Y, Zhao M, Li Y-Q, Sun ZS (2012) Regulation of peripheral clock to oscillation of substance $P$ contributes to circadian inflammatory pain. Anesthesiology 117:149-160

Zhong J, Liang M, Akther S, Higashida C, Tsuji T, Higashida H (2014) c-Fos expression in the paternal mouse brain induced by communicative interaction with maternal mates. Mol Brain 7:66. 